\title{
Nesprin interchain associations control nuclear size
}

\author{
Wenshu Lu • Maria Schneider • Sascha Neumann • Verena-Maren Jaeger • \\ Surayya Taranum • Martina Munck - Sarah Cartwright - Christine Richardson • \\ James Carthew · Kowoon Noh • Martin Goldberg • Angelika A. Noegel • \\ Iakowos Karakesisoglou
}

Received: 20 November 2011 / Revised: 26 April 2012 / Accepted: 14 May 2012 / Published online: 1 June 2012

(C) Springer Basel AG 2012

\begin{abstract}
Nesprins-1/-2/-3/-4 are nuclear envelope proteins, which connect nuclei to the cytoskeleton. The largest nesprin-1/-2 isoforms (termed giant) tether F-actin through their N-terminal actin binding domain (ABD). Nesprin-3, however, lacks an ABD and associates instead to plectin, which binds intermediate filaments. Nesprins are integrated into the outer nuclear membrane via their C-terminal KASHdomain. Here, we show that nesprin-1/-2 ABDs physically and functionally interact with nesprin-3. Thus, both ends of nesprin-1/-2 giant are integrated at the nuclear surface: via the C-terminal KASH-domain and the N-terminal ABD-nesprin3 association. Interestingly, nesprin-2 ABD or KASHdomain overexpression leads to increased nuclear areas. Conversely, nesprin-2 mini (contains the ABD and KASHdomain but lacks the massive nesprin-2 giant rod segment) expression yields smaller nuclei. Nuclear shrinkage is further
\end{abstract}

Electronic supplementary material The online version of this article (doi:10.1007/s00018-012-1034-1) contains supplementary material, which is available to authorized users.

W. Lu · M. Schneider · V.-M. Jaeger · S. Cartwright .

C. Richardson - J. Carthew · K. Noh · M. Goldberg .

I. Karakesisoglou $(\square)$

School of Biological and Biomedical Sciences,

University of Durham, Durham DH1 3LE, UK

e-mail: iakowos.karakesisoglou@durham.ac.uk

W. Lu · M. Schneider · S. Neumann · V.-M. Jaeger ·

S. Taranum - M. Munck $\cdot$ A. A. Noegel

Center for Biochemistry, Medical Faculty, University of

Cologne, Joseph-Stelzmann-Strasse 52, 50931 Cologne,

Germany

\section{A. A. Noegel}

Center for Molecular Medicine Cologne, Medical Faculty, University of Cologne, Joseph-Stelzmann-Strasse 52, 50931

Cologne, Germany enhanced upon nesprin-3 co-expression or microfilament depolymerization. Our findings suggest that multivariate intermolecular nesprin interactions with the cytoskeleton form a lattice-like filamentous network covering the outer nuclear membrane, which determines nuclear size.

Keywords Actin binding domain - Cytoskeleton · KASH-domain · LINC complex · Nesprin · Nuclear envelope $\cdot$ Nuclear shape $\cdot$ SUN-domain

$\begin{array}{ll}\text { Abbreviations } \\ \text { aa } & \text { Amino acid } \\ \text { ab } & \text { Antibody } \\ \text { ABD } & \text { Actin binding domain } \\ \text { DAPI } & 4^{\prime}, 6 \text {-diamidino-2-phenylindole } \\ \text { ER } & \text { Endoplasmic reticulum } \\ \text { GFP } & \text { Green fluorescent protein } \\ \text { GST } & \text { Glutathione S-transferase } \\ \text { KASH } & \text { Klarsicht, ANC-1, and Syne Homology } \\ \text { INM } & \text { Inner nuclear membrane } \\ \text { LINC } & \text { Linker of the nucleoskeleton and cytoskeleton } \\ \text { NE } & \text { Nuclear envelope } \\ \text { Nesprin } & \text { Nuclear envelope spectrin repeat protein } \\ \text { ONM } & \text { Outer nuclear membrane } \\ \text { PNS } & \text { Perinuclear space } \\ \text { phall } & \text { Phalloidin } \\ \text { siRNA } & \text { Small interfering RNA } \\ \text { SR } & \text { Spectrin repeat } \\ \text { SUN } & \text { Sad1 and UNC-84 }\end{array}$

\section{Introduction}

The nuclear envelope (NE) is the defining feature of all eukaryotes, which separates the nucleoplasm from the 
cytoplasm. It is composed of two structurally distinct and concentric lipid bilayers, the inner and outer nuclear membranes (INM and ONM). These are separated by the perinuclear space (PNS), connected at nuclear pore complexes (NPCs) and bridged by specific ONM-INM protein assemblies (termed the LINC complex), that connect cytoplasmic structures with the nuclear interior [1-3]. The ONM is contiguous with the rough endoplasmic reticulum (ER), while the INM contains distinct membrane proteins, which form close associations with the underlying chromatin and nuclear lamina. The lamina provides a structural scaffold to the NE and is composed primarily of nuclear intermediate filament proteins, the lamins [4-7].

LINC complex components have been found in a wide array of organisms that include plants, yeast, amoebae, worms, flies, and vertebrates [8-13]. The complex is composed of the ONM-resident KASH (Klarsicht, ANC-1, and Syne Homology) and the INM-targeted SUN (Sad1 and UNC-84) domain proteins. The C-terminal KASHdomain encompasses a single transmembrane segment, which is followed by an evolutionarily conserved PNSsituated short peptide that associates directly with SUN protein luminal domains [14-19]. Mammalian SUNdomain proteins form stable oligomeric assemblies and are essential for KASH-domain protein recruitment to the ONM [14, 20]. In contrast to their highly conserved C-termini, KASH protein N-terminal segments are divergent. There is considerable variation in length, due to differing spectrin repeat (SR) copy numbers. They also contain specific domains, which interact with different proteins, enabling the integration of the ONM with various cytoskeletal structures $[12,21,22]$.

To date, four proteins with KASH-domains and SRs have been identified in mammals, termed nesprins-1/-2/-3/4. Each nesprin is encoded by a separate gene that gives rise to a multitude of structurally and functionally diverse isoforms [23-27]. The largest gene products of the syne-1/2 loci are referred to as nesprins-1/-2 giant (alternatively known as NUANCE and Enaptin, respectively). These macromolecules $(>800 \mathrm{kDa})$ comprise an N-terminal actin binding domain (ABD), which is separated from the C-terminal nuclear membrane integration site by a massive SR rod segment [24, 25]. In contrast, nesprin-3 and -4 proteins are much smaller $(<116 \mathrm{kDa})$ and lack an ABD. It is noteworthy, however, that nesprin-3 possesses an $\mathrm{N}$-terminal binding site for the plectin $\mathrm{ABD}$, which is a versatile cytolinker that crossbridges all major cytoskeletal elements including intermediate filaments [26, 28]. Moreover, the tissue-specific nesprin-4 associates to the plus-end microtubule motor kinesin-1 and contributes to cellular polarization [27]. Thus, nesprins are key and versatile determinants of the ONM cytoskeletal landscapes, which define cellular architecture and cell behavior. These molecules endow the mammalian cell with a diverse repertoire of fundamental biological functions. Nesprins have roles in the control of cellular stiffness, ciliogenesis, organelle positioning (e.g., nucleus, Golgi, centrosome), endocytosis, Wnt-signaling, directed cellular migration, and cell adhesion [12, 22, 29-38].

Human and murine genetic studies further underline their biological significance and functional diversity at the organismal level. Nesprin-1 and -2 defects have been linked to human diseases including: muscular dystrophy, arthrogryposis, ataxia, progeria, lissencephaly, and cancer [39-46]. Nesprin-1/-2 KASH-domain double knockout mice die shortly after birth due to respiratory failure [31]. However, single nesprin-1 C-terminal knockouts vary in their phenotypes depending on which exons are deleted. Overall, nesprin-1 mouse mutants exhibit decreased survival rates, severe growth retardation, kyphoscoliosis, neurogenesis defects, and skeletal/cardiac muscle pathologies [31, 47, 48]. In contrast, nesprin-2 KASH-domain knockout mice are viable, but display severe learning and memory deficits [35]. Similarly, nesprin-2 giant-deficient mice are also viable and do not exhibit any gross physical abnormalities. However, the loss of nesprin-2 giant impairs NE architecture morphology and composition [32, 41]. As a consequence, nuclei become irregularly shaped and enlarged, which contributes to a mild increase in epidermal thickness [32]. How nesprin-2 giant controls nuclear shape remains largely unresolved. Current models depict the giant nesprin-1/-2 KASH-domain isoforms as radiating ONM molecules projecting into the cytoplasm. These massive molecules are conceived as flexible spokes that function as the suspension apparatus of the nucleus rather than rigid molecules that encase the nuclear exterior. Clearly, the former arrangement does not accommodate well the structural defects that occur when these massive proteins are missing [32, 42]. More importantly, all currently available models are speculative and have not been experimentally validated.

In the current paper, we provide compelling evidence that nesprin-1/-2 giant proteins are integrated at the NE, at both ends of the molecules. While the KASH-domain embeds the C-terminus directly into the ONM, molecular interactions that involve nesprin-3 and the nesprin-1/-2 N-termini may account for their alignment along the nuclear surface. In agreement with this model, interference with nesprin-2 giant $\mathrm{N}$ - or C-terminal domain binding results in nuclear expansion. Conversely, the co-expression of nesprin-3 and nesprin-2 fusions, that lack the massive central rod domain of nesprin-2 giant, triggers nuclear shrinkage. Taken together, these data suggest the presence of a filamentous nesprin-based meshwork, which structurally supports and links the outer nuclear surface to its cellular surrounding. 


\section{Materials and methods}

Cloning strategies and siRNA approaches

GFP-Nesprin-1 ABD (aa 2-296) and GFP-Enaptin-165 (aa 1-1,431) are described elsewhere [25]. GFP-Nesprin-2 KASH is equivalent to TmNesprin-2 (aa 6,835-6,885) [49]. GFP-Nesprin-2 ABD (aa 1-285) and GFP-Nesprin-2 mini (aa 1-459, 6,644-6,885) are equal to GFP-ABD and GFP$\mathrm{NUA}^{\Delta 460-6,643}$, respectively [24]. Nesprin-2 SR-KASH was amplified via RT-PCR from HaCaT cDNA and ligated into the EcoRI/SalI cloning site of EGFP-C2. Nesprin-2 SR-KASH equals the aa $6,147-6,885$ sequence of Nesprin-2 giant, except that it contains a 23 aa insertion (DVEIPENPEAYLKMTTKTLKASS, which corresponds to an alternative spliced exon) after aa 6,444. Human Nesprin-3 full-length (FL) (aa 1-975; cDNA inserted into the KpnI/NotI cloning site of pCMV-HA, or into the KpnI/ NotI cloning site of pCMV-Myc); Nesprin-3 $\Delta \mathrm{C}$ (aa 1-441; inserted into the AhdI/SacII cloning site of EGFP-C2); Nesprin-3 KASH (aa 924-975; inserted into the KpnI/NotI cloning site of pCMV-Myc); Nesprin-3 SR1, Nesprin-3 SR2, Nesprin-3 SR1,2 and Nesprin-3 SR1,2,3 (aa 1-106, aa 104-228, aa 1-228 and aa 1-325, respectively; cloned into EcoRI/SalI digested pGEX4T1) were constructed by PCR using the AK098471 and AK131436 cDNAs (Department of Biotechnology, Japan, http://www.nbrc.nite.go.jp) as templates. GFP-Plectin-1c ABD was kindly provided by Dr. Arto Maatta.

siRNA experiments were performed according to the manufacturer's instructions (Qiagen). The following siRNAs were used: FlexiTube Hs_PLEC_7 targeting the human plectin sequence 5'-CAAGGTGTACCGGCAGA CCAA-3' and AllStars negative control duplexes (all from Qiagen). In brief, cells were allowed to recover for 2 days after the first siRNA treatment, before they were subjected to a second round of siRNA tranfection. The following day, cells were seeded on glass cover slips, fixed with methanol the next day and processed for immunofluorescence staining.

Cell culture, cytoskeleton pharmacological treatments and transfections

COS7, human keratinocyte (HaCaT) and human primary fibroblasts were grown at $37{ }^{\circ} \mathrm{C}, 5 \% \mathrm{CO}_{2}$ in high glucose DMEM (Sigma-Aldrich) supplemented with $2 \mathrm{mM}$ glutamine, $1 \%$ penicillin/streptomycin and $10 \%$ FBS.

COS7 cells were transiently transfected at $170 \mathrm{~V}, 950$ $\mu \mathrm{F}$ by electroporation using a Gene-Pulser (Bio-Rad). For transient transfections of the $\mathrm{HaCaT}$ cell line, the Amaxa Cell Line Nucleofector Kit V (Lonza) was utilized. Transfected cells were grown for $24-48 \mathrm{~h}$ before fixation.
To depolymerize F-actin, cells were treated with latrunculin B ( $1 \mu \mathrm{g} / \mathrm{ml}$; Sigma-Aldrich) for $30 \mathrm{~min}$ (COS7 cells) or $60 \mathrm{~min}$ (HaCaT cells), before fixation. To depolymerize microtubules, cells were treated with $12 \mu \mathrm{M}$ colchicine (Sigma-Aldrich) for $60 \mathrm{~min}$ before fixation.

Antibodies and immunofluorescence (IF) microscopy

Cells grown on cover slips were fixed in $4 \%$ paraformaldehyde in phosphate-buffered saline (PBS) for $10 \mathrm{~min}$ followed by permeabilization with $0.5 \%$ Triton X-100 for 5 min. Alternatively, cells were fixed in cold methanol $\left(-20^{\circ} \mathrm{C}\right)$ for $10 \mathrm{~min}$. Fixed samples were blocked in phosphate-buffered gelatine (PBG; contains $0.1 \%$ cold water fish-gelatine and $0.5 \% \mathrm{BSA}$ ) for $15 \mathrm{~min}$ and incubated with primary antibodies (diluted in PBG) for $60 \mathrm{~min}$ at room temperature. The following antibodies were used: GFPspecific mAb K3-184-2 [50], mouse anti-myc [51], mouse anti-cytokeratin (AE1/AE3; Millipore), mouse anti- $\beta$-actin (AC-74; Sigma-Aldrich), mouse anti-tubulin (WA3; kind gift of Dr. U. Euteneuer), mouse anti-vinculin (hVIN-1; Sigma-Aldrich), rabbit anti-GST [20], rat anti-HA (3F10; Santa Cruz Biotechnology), goat anti-plectin (C-20; Santa Cruz Biotechnology), rabbit anti-plectin (Abcam), mouse monoclonal anti-nesprin-3 (kind gift of Dr. A. Sonnenberg; [26]), mouse anti-nes2NT (mAb K20; [24]), affinity-purified rabbit anti-nes2CT (pAb K1; [49]), and rabbit anti-nes1NT and anti-nes1CT [25]. Cells were extensively washed in PBS and then incubated with the relevant secondary antibodies, which were conjugated with Cy5 (Sigma-Aldrich), Alexa Fluor 488, Alexa Fluor 568, or Alexa Fluor 647 (Invitrogen). PBS-washed specimens were then mounted in Gelvatol/ DABCO (Sigma-Aldrich). F-actin was visualized by TRITC-labelled phalloidin (Sigma-Aldrich) and DNA was counterstained with 4',6-diamino-2-phenylindone (DAPI; Sigma-Aldrich). All samples were analyzed by confocal laser-scanning microscopy using either a TCS-SP1/SP5 (Leica) or a LSM510-Meta (Zeiss).

Purification of GST fusion proteins, GST pull-down, His pull-down assays, western blotting and immunoprecipitation

GST-fusion proteins (GST-nes-3 SR1, GST-nes-3 SR2, GST-nes-3 SR1,2, and GST-nes-3 SR1,2,3 fusions, respectively) were bound to Glutathione-SepharoseTM 4B (Amersham) at $4{ }^{\circ} \mathrm{C}$ overnight. GFP-nes-1 ABD, GFP-nes2 ABD, or GFP-plectin-1c ABD transfected COS7 cell lysates were generated using lysis buffer $(50 \mathrm{mM}$ Tris/HCl, pH 7.5, $150 \mathrm{mM} \mathrm{NaCl}, 1 \%$ Nonidet P-40, $0.5 \%$ sodium deoxycholate and protease inhibitors; Roche). The lysates were then incubated with equal quantities of GST-fusion proteins coupled to GST-Sepharose beads at $4{ }^{\circ} \mathrm{C}$ 
overnight. Samples were centrifuged and pellets were extensively washed with PBS. Supernatant and pellet samples were assessed by SDS-polyacrylamide gel electrophoresis (SDS-PAGE) concomitant by Coomassie Blue staining and western blot analysis [52]. For His pull-down assays, E. coli expressed His-nesprin-1 ABD and His-nesprin-2 ABD were coupled to Ni-NTA agarose beads by incubation for $4 \mathrm{~h}$ at $4{ }^{\circ} \mathrm{C}$ as described in the manufacturer's manual (Qiagen). GFP or GFP-nesprin-3 $\Delta \mathrm{C}$ transfected COS7 lysates were incubated with beads coupled with His-fusions at $4{ }^{\circ} \mathrm{C}$ overnight. The pellets were washed with PBS and analyzed by western blot. For immunoprecipitation analysis, myc-nesprin-3 full-length transfected COS7 cells were lysed in buffer containing $50 \mathrm{mM}$ Tris-HCl, pH 7.5, $150 \mathrm{mM} \mathrm{NaCl}$, and $0.5 \%$ Nonidet P-40, and the immunoprecipication was performed as described [49].

\section{F-actin binding assays}

Purified skeletal muscle actin (Cytoskeleton, \#AKL99) was resuspended in G-buffer $(0.2 \mathrm{mM} \mathrm{CaCl} 2,0.2 \mathrm{mM}$ ATP, $0.5 \mathrm{mM}$ DTT, $5 \mathrm{mM}$ Tris, $\mathrm{pH} 8.0$ ), incubated for $1 \mathrm{~h}$ on ice and subjected to centrifugation at $30,000 \mathrm{~g}$ for $15 \mathrm{~min}$ at $4{ }^{\circ} \mathrm{C}$, to remove actin polymers. His-tagged nes- $2 \mathrm{ABD}$ was purified as described above, concentrated using Pierce protein concentrators (20K MWCO; Thermo Scientific), and dialyzed overnight at $4{ }^{\circ} \mathrm{C}$ in P-buffer $\left(2 \mathrm{mM} \mathrm{MgCl}_{2}\right.$, $50 \mathrm{mM} \mathrm{KCl}, 1 \mathrm{mM}$ ATP, $0.5 \mathrm{mM}$ DTT, $5 \mathrm{mM}$ Tris, $\mathrm{pH}$ 8.0) containing protease inhibitors. Glutathione-Sepharose coupled GST-nes-3 SR1,2,3 or GST proteins were incubated with His-tagged nes- 2 ABD in the absence or presence of actin for $1 \mathrm{~h}$ at room temperature, under conditions that induce F-actin polymerization (usage of P-buffer). The samples were centrifuged at $1,000 \mathrm{~g}$ for 2 min and the pellets (PD: pull-down) were prepared for SDS-PAGE analysis while the supernatants were ultracentrifuged at $100,000 \mathrm{~g}$ for $60 \mathrm{~min}$ at $4{ }^{\circ} \mathrm{C}$. The resulting supernatants $(\mathrm{S})$ and pellets $(\mathrm{P})$ were mixed with Laemmli sample buffer and resolved by SDS-PAGE together with the PD samples and then stained by Coomassie Blue to visualize the proteins.

\section{Statistical and morphometric analysis}

Statistical analysis was performed using Student's $t$ test, and data are shown as mean $(\%) \pm \mathrm{SD}$ (standard deviation) unless otherwise stated. $P$ values $\leq 0.05$ (denoted with *; decimal decrements indicated by the addition of further $*$ ) were considered significant.

HaCaT cells (2 days post-transfection) were imaged using a Leica SP5 confocal microscope. The mean nuclear area of transfected cells was measured (largest cross- sectional nuclear area) using the software Image $\mathrm{J}$, and normalized by the mean nuclear area of untransfected HaCaT cells (200 cells computed for each experiment; except for the SR-KASH and SR-KASH and nes-3 FL transfections where at least 60 nuclei were analyzed; Fig. 7f). Alternatively, mock and GFP-fusion transfected HaCaT cells were subjected (either directly or incubated for $1 \mathrm{~h}$ with cytoskeleton depolymerizing drugs prior fixation) to immunofluorescence and DAPI staining 2 days after transfection and imaged using an Axioskop 40 Zeiss epifluorescence microscope. Images were recorded with an AxioCam MRM (Zeiss) and the cell (200 phalloidinstained cells counted for each condition; Fig. $7 \mathrm{~g}$ ) and nuclear area (100 cells counted for each condition; Fig. 8e) were calculated using the AxioVision v.4.5 software (Zeiss). All cells were plated at identical cell densities and only transfected cells situated at the periphery of small colonies ( $2-8$ cells) were measured.

\section{Results}

Nesprin-3 full-length proteins display reduced dominant negative properties compared to nesprin-3 KASH-domain fusions

To gain insights into the NE targeting mechanisms of nesprins, we examined the KASH-domain localization properties of the widely expressed nesprin-1/-2/-3 paralogues. We did not include nesprin-4, because of its confined expression in secretory epithelial cells. Since nesprins-1/-2 are differentially expressed, $\mathrm{HaCaT}$ and COS7 are our preferred cellular models for nesprin-2 studies, while fibroblasts are employed to investigate NEassociated nesprin-1 isoforms.

As indicated in Fig. 1a, nesprin KASH-domains are highly homologous to each other. Similar to other nesprins and in agreement with previous reports [28], when the myc-tagged nesprin-3 KASH-domain (termed nes-3 KASH; see Fig. S1 for structural details) was expressed, it localized specifically to the NE (Fig. 1b, arrows). When overexpressed, it affected the endogenous nesprin-2 staining pattern in $\mathrm{HaCaT}$ keratinocytes (Fig. $1 b^{\prime}$, c, arrows) or nesprin-1 in fibroblasts (Fig. S2A-A $\mathrm{A}^{\prime \prime \prime}$ ). Specifically, in 71 and $76 \%$ of $\mathrm{HaCaT}$ cells expressing myc-tagged nes-3 KASH (Fig. 1d), nesprin-2 NE staining was not detectable using either nesprin-2 giant C-terminal (Fig. 1b', arrows) or N-terminal (Fig. 1c, arrows) epitope-directed antibodies, respectively. Only untransfected cells displayed nesprin-2 at the NE (Fig. 1b' c, arrowheads). Conversely, nesprin-2 KASH (termed nes-2 KASH; Fig. S1) overexpression affected the localization of co-transfected HA-tagged fulllength nesprin-3 $\alpha$ (termed nes-3 FL, Fig. S1) in $96 \%$ of 

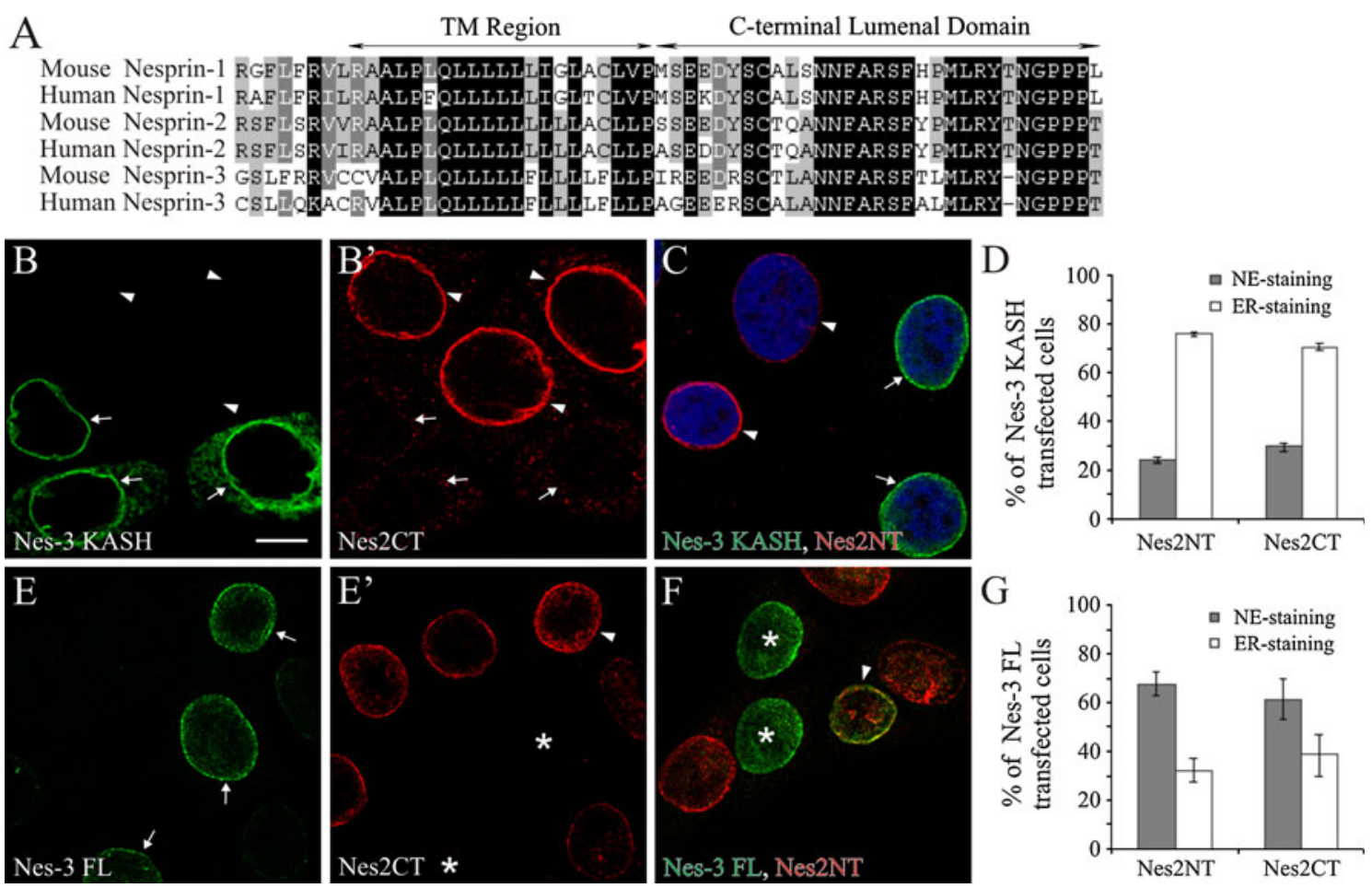

Fig. 1 a-g Nesprin C-termini are conserved and tethered at the NE by an identical mechanism. a Sequence alignment of mouse and human nesprin-1/-2/-3 KASH-domains, identical amino acids are depicted in black and similar residues in gray. $\mathbf{b}, \mathbf{b}^{\prime}$ The expression of myc-tagged nesprin-3 (nes-3) KASH fusions exerts dominant negative effects on endogenous nesprin-2 proteins. Transiently transfected $\mathrm{HaCaT}$ cells were subjected to immunofluorescence using nesprin-2 C-terminal (Nes2CT) and anti-myc antibodies. Note the prominent nuclear rim staining of endogenous nesprin-2 in untransfected cells $\left(\mathbf{b}^{\prime}\right.$, arrowheads) and the absence of nesprin-2 staining in mycpositive cells ( $\mathbf{b}^{\prime}$, arrows). c Myc-tagged nes-3 KASH positive $\mathrm{HaCaT}$ cells subjected to immunofluorescence using nesprin-2Nterminal (Nes2NT) antibodies lack also nesprin-2 staining at the NE (arrows). d Histogram representing the nes-3 KASH displacement

transfected cells (Fig. S2B-B' ${ }^{\prime \prime \prime}$ ). Together, these data confirm previous studies, which demonstrated the promiscuous tethering of nesprin C-termini at the $\mathrm{NE}$ by a common SUN-domain protein-based mechanism [28, 33]. Essentially, excess KASH-domains compete for SUN protein interactions with endogenous KASH-domain proteins. In contrast to the dramatic dominant negative effects of nes-3 KASH on nesprin-2, nesprin-2 was more resilient to NE dislodgement when nesprin-3 $\alpha$ full-length (nes-3 FL) was employed. A statistical analysis showed that 61 and $68 \%$ of cells transiently expressing HA-tagged nes-3 FL (Fig. 1g) still retained C-terminal (Fig. 1e' ${ }^{\prime}$, arrowhead) and $\mathrm{N}$-terminal (Fig. 1f, arrowhead) nesprin-2 antibody staining, respectively. Thus, overexpression of just the KASHdomain had a much greater affect on nesprin-2 than the overexpression of the complete nesprin-3 protein. This result raised the question of whether nesprin- $3 \alpha$ could directly interact with nesprin-2. effects on the localization of endogenous nesprin-2 from the NE to the ER (endoplasmatic reticulum) in transfected HaCaT cells (300 transfected cells each were evaluated). e-f HA-tagged nes-3 FL transiently transfected $\mathrm{HaCaT}$ cells processed for immunofluorescence using anti-HA and either nesprin-2 C-terminal (Nes2CT, e, $\left.\mathbf{e}^{\prime}\right)$ or N-terminal antibodies (Nes2NT, f). Note that although nes-3 FL localized to the NE (e, arrows), its effects on nesprin-2 are heterogeneous. Some cells lack nesprin-2 (é⿱ ${ }^{\prime}, \mathbf{f}$, asterisks) whereas some still retain staining at the NE ( $\mathbf{e}^{\prime}, \mathbf{f}$, arrowheads). DNA was stained with DAPI. Scale bar $10 \mu \mathrm{m}$. g Statistical evaluations of the diminished dominant negative effects of full-length nesprin-3 fusions on endogenous nesprin-2 (examples shown in $\mathbf{e}-\mathbf{f} ; 300$ cells counted for each experiment)

Ectopically expressed nesprin-3 recruits the nesprin-2 $\mathrm{ABD}$, which in turn recruits F-actin

Nesprin-3 associates in vitro with the ABDs of the cytolinker proteins plectin and MACF1 (microtubule-actin cross-linking factor 1) [26]. This suggested that there might also be interactions between nesprin-3 and the ABDs of nesprins-1 and -2 . This assumption was based exclusively on the high protein sequence homologies shared among the plectin, MACF1 and nesprin-1/-2 giant ABDs (Fig. S3; [26]). For example, the plectin ABD displays 42.75 and $43.53 \%$ identity to the nesprin- 1 and nesprin-2 ABDs, respectively. To evaluate whether the nesprin-1/-2 ABDs indeed interact with nesprin-3, we first examined their localization pattern by transfection studies. The experiments were executed in COS7 cells where nesprin-3 expression is low [26] and largely absent from the NE (Fig. S4B'). In agreement with previous work [24], GFP-tagged 
nesprin-2 ABD alone (nes-2 ABD; Fig. S1) predominantly colocalized with actin microfilaments as shown by TRITCphalloidin staining in transfected cells (Fig. 2a, b-b", arrowheads). However, in a proportion of cells (12.5\%), we noticed weak GFP-nes-2 ABD NE staining (Fig. 2b, arrows, g).

Similar to HaCaT cells (Fig. 1e), ectopically expressed nes-3 FL accumulated prominently at the nuclear rim of COS7 transfected cells (Fig. 2c, d). The overexpression of nesprin- $3 \alpha$ alone did not trigger significant F-actin recruitment around the nucleus (Fig. 2c). When GFP-nes-2 ABD was co-expressed with nes-3 FL, it resulted in a dramatic accumulation of nes-2 ABD at the nuclear surface (Fig. 2d $\mathrm{d}^{\prime}, \mathrm{d}^{\prime \prime}$, arrow) in $82 \%$ of transfectants (Fig. $2 \mathrm{~g}$ ). Interestingly, the accumulation of nes-2 ABD at the NE coincided now with prominent peri-nuclear F-actin structures (Fig. 2e-e $\mathrm{e}^{\prime \prime \prime}$ ). Since nesprin-3 has the ability to redistribute plectin [26], which is an F-actin binding protein, the nesprin-2 ABD relocation may have emerged as the result of plectin-mediated recruitment of ABD-decorated actin structures to the nuclear rim, as opposed to direct associations between nesprins-2 and -3 . To investigate this caveat, we treated cells that were co-expressing nesprin-2 ABD and nesprin-3 with latrunculin $\mathrm{B}$, which prevents actin polymerization. Similar to untreated cells, nesprin-3 still recruited the nesprin-2 ABD to the NE while F-actin was absent (Fig. $2 \mathrm{f}-\mathrm{f}^{\prime \prime \prime}$ ). Thus, the nesprin-3 induced recruitment of the nesprin-2 ABD is not mediated via F-actin.

Nesprin-3 associates in vitro with the nesprin-1/-2 ABDs

We next examined biochemically the association of nesprin-3 with the nesprin-1 and nesprin-2 ABDs using Histagged protein pull-down assays. We also included the nesprin-1 ABD in our analysis (nes-1 ABD; Fig. S1) because of its high primary sequence identity to nesprin-2 (53.49 \%; Fig. S3), which suggested that nesprin-1 and -2 ABDs may display similar biochemical properties with respect to nesprin-3. For this analysis, we used nesprin-3 $\Delta \mathrm{C}$, which is a $\mathrm{C}$-terminal truncated construct, that harbors only the first four nesprin- $3 \alpha$ spectrin repeats (SR) and which lacks the KASH-domain (see Fig. S1 for structural details). Although only the first nesprin-3 SR is required for plectin binding, we decided to employ a longer nesprin-3 SR fusion (nesprin-3 $\Delta \mathrm{C}$ ) in order to facilitate proper SR folding. As anticipated, both Histagged nesprin-1 and nesprin-2 recombinant ABDs specifically precipitated GFP-tagged nesprin-3 $\Delta \mathrm{C}$ and not GFP alone (Fig. 2h). Overall, these results show that nesprin-3 can specifically interact with the ABDs of nesprins- 1 and -2 .
Fig. 2 a-h Nesprin-3 associates with the nesprin-1/-2 ABDs in vitro and recruits the nesprin-2 ABD to the NE. a Immunofluorescence of GFP-tagged nesprin-2 (nes-2) ABD transfected COS7 cells stained with TRITC-phalloidin (Phall; reveals F-actin), indicates that the nesprin-2 ABD colocalizes with F-actin. $\mathbf{b}-\mathbf{b}^{\prime \prime}$ In a small number of GFP-tagged nes-2 ABD transfected COS7 cells the nesprin-2 ABD localizes at the NE (b,arrows); colocalization with actin filaments is also indicated (b-b' $\mathbf{b}^{\prime \prime}$, arrowheads). $\mathbf{c}$ In HA-tagged nes-3 FL (nes-3 $F L)$ transfected COS7 cells, pronounced HA-staining is found at the NE, which does not colocalize with phalloidin. d-d $\mathbf{d}^{\prime \prime}$ In most HAtagged nes-3 FL and GFP-tagged nes-2 ABD co-transfected COS7 cells, the GFP-fusion decorated intensively the NE and colocalized with nesprin-3. $\mathbf{e}-\mathbf{e}^{\prime \prime \prime}$ Phalloidin staining of HA-tagged nes-3 FL and GFP-tagged nes-2 ABD co-transfected cells, reveals prominent F-actin structures at the NE. $\mathbf{f}-\mathbf{f}^{\prime \prime \prime}$ Similarly, co-transfected cells were treated with latrunculin B. F-actin disruption (absence of phalloidin stain, $\mathbf{f}^{\prime \prime}$ ) did not affect the persistence of nes-2 ABD at the nuclear surface $\left(\mathbf{f}^{\prime}\right)$. Scale bars $10 \mu \mathrm{m}$. g Histogram representing a statistical evaluation (percentage of transfected cells, $n=300$ ) of the GFP-tagged nes-2 ABD localization profiles to the cytoplasm (Cyto) and the $N E$, in single and in HA-tagged nes-3 FL co-transfected COS7 cells. h COS7 cell lysates expressing GFP-tagged nesprin-3 $\Delta \mathrm{C}$ (GFP-nes-3 $\triangle C$ ) were incubated with immobilized His-tagged fusion proteins (His-nes-1 ABD, His-nes-2 ABD) as indicated. COS7 cell lysates expressing GFP alone were used as control. The cell lysates (Input) and the specifically bound proteins (Pellet) were separated by SDS-PAGE and subjected to western blot analysis using GFP specific antibodies (upper panels) or were directly stained with Coomassie Blue to demonstrate equal recombinant protein usage (lower panel)

Nesprin-3 recruits nesprin-1 ABD-containing isoforms to the NE

Utilizing HaCaT keratinocytes and COS7 cells, we next examined the effects of nesprin-3 overexpression on endogenous nesprin- 1 proteins. We used $\mathrm{HaCaT}$ cells because previous studies, using ABD-directed antibodies (epitope indicated in Fig. S1 as Nes1NT) in epidermal keratinocytes, indicated an almost exclusive cortical distribution of endogenous nesprin-1 [32]. As expected, the antibodies detected nesprin-1 along cortical F-actin based structures (Fig. 3a-a $\mathrm{a}^{\prime \prime \prime}$, arrowheads). A faint nesprin-1 NE localization was evident only in a minor proportion of keratinocytes (7.5\%; Fig. 3E). However, when HA-tagged nes-3 FL was transiently expressed, nesprin-1 was efficiently relocated to the NE (Fig. 3b, arrows) and strong nesprin-1 NE rim staining was evident in $78 \%$ of nesprin$3 \alpha$ positive cells (Fig. 3e). Strikingly, the nesprin-1 relocation to the NE coincided largely with a repositioning of F-actin structures to the nuclear surface (Fig. $3 b^{\prime}$, arrows).

To allow further insights into the nesprin-3 mediated recruitment mechanism, we analyzed COS7 cells, which enable high transfection rates, and looked at the subcellular distribution of ectopically expressed GFP-tagged Enaptin165 , an N-terminal ABD-containing isoform of nesprin-1 (Fig. S1). As previously reported [25], when GFP-Enaptin165 alone was ectopically expressed, it localized to F-actin rich structures (e.g., cortical regions and stress-fibres) 

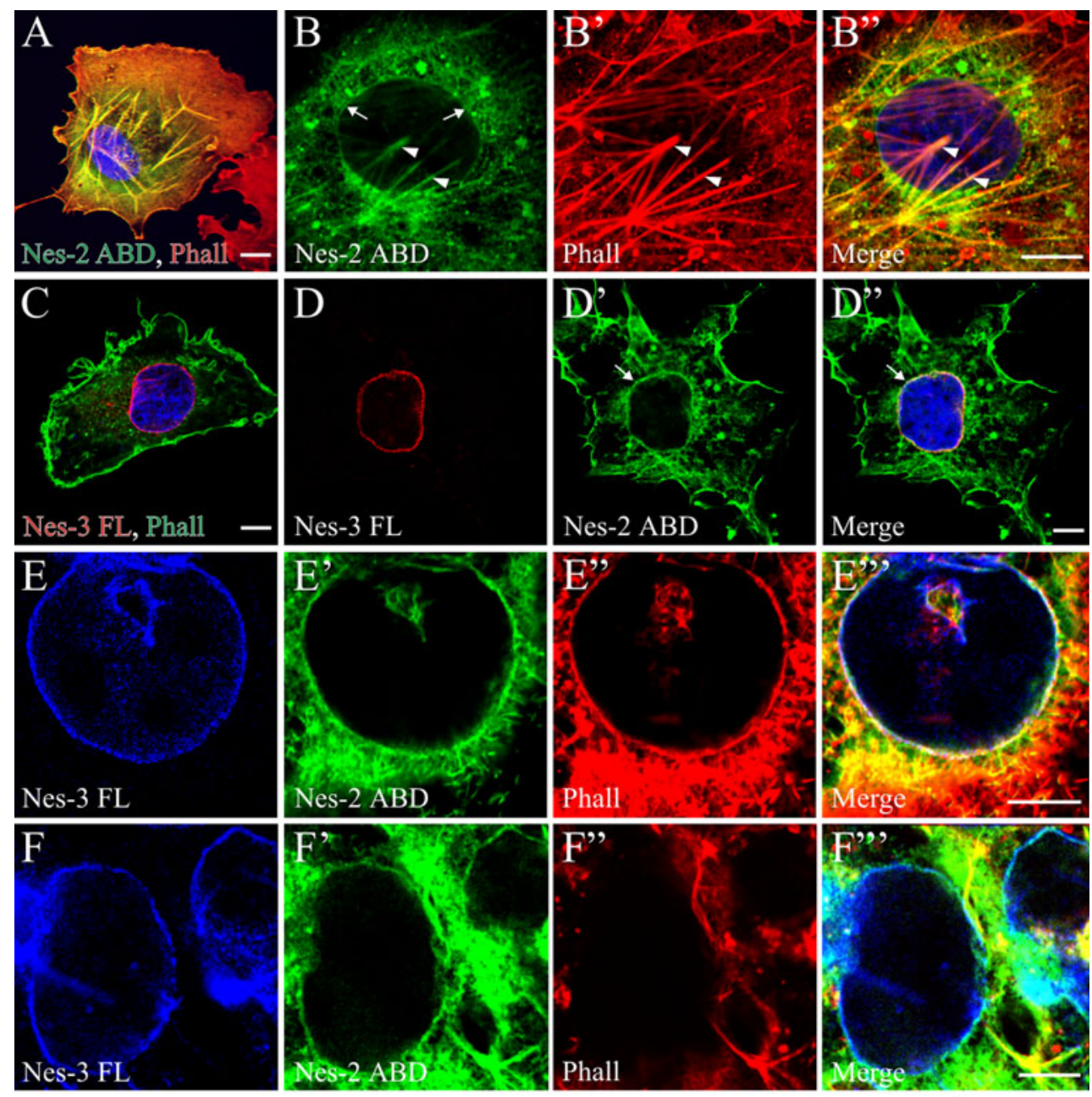

G

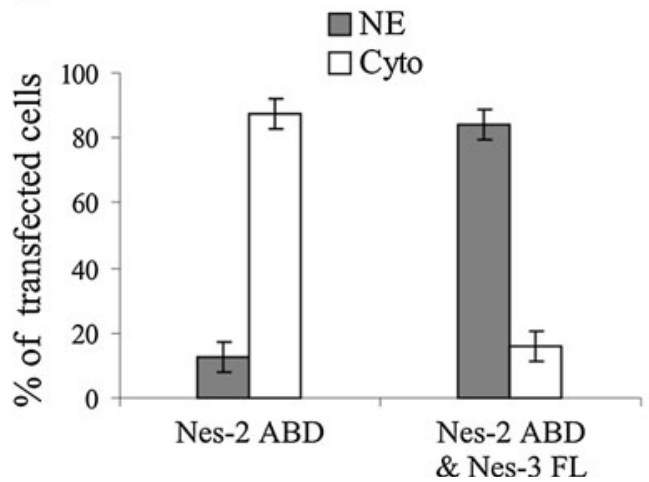

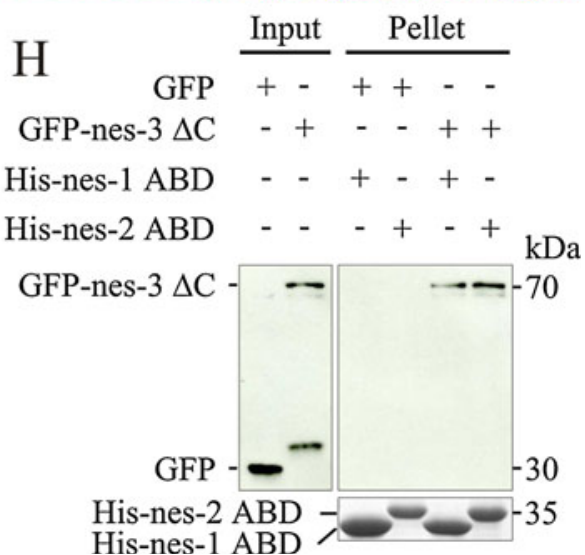

The nesprin-2 ABD interacts directly with nesprin-3 in the presence of F-actin

To provide further insights into the association of nesprin-2 ABD with nesprin-3 and F-actin, we next assessed these interactions biochemically in vitro. For this purpose, we performed GST pull-downs (PD) using either GST (negative control) or GST-nesprin-3 SR1,2,3 (contains the first three spectrin repeats of nesprin-3 $\alpha$; see Fig. S1 for 
Fig. 3 a-f Nesprin-3 recruits nesprin-1 ABD-containing isoforms to the NE. $\mathbf{a}-\mathbf{a}^{\prime \prime \prime}$ Endogenous nesprin-1 colocalizes with F-actin in HaCaT cells. The cells were stained with nesprin-1 ABD directed antibodies (nes1NT; Fig. S1) and TRITC-phalloidin (Phall). b-b ${ }^{\prime \prime \prime}$ HaCaT cells transiently expressing HAtagged nes-3 FL were processed for immunofluorescence using anti-HA and nesprin-1 (nes1NT) antibodies. Endogenous nesprin-1 colocalizes with both nesprin-3 and F-actin at the NE (arrows). c, $\mathbf{c}^{\prime}$ Ectopically expressed GFP-tagged Enaptin165 localizes to actin-based structures (arrowheads) in COS7 cells. d, d' In GFPEnaptin-165/HA-nes-3 FL cotransfected cells, GFP-Enaptin165 was recruited to the NE (arrows). Scale bars $10 \mu \mathrm{m}$. e, f Histograms representing the percentage of cells exhibiting (gray bars) or lacking (white bars) NE-staining: for endogenous nesprin-1 (e) in untransfected (Control) or HAnes-3 FL transfected COS7 cells and for GFP-Enaptin-165 (f) in single or HA-nes-3 FL double transfected cells, respectively
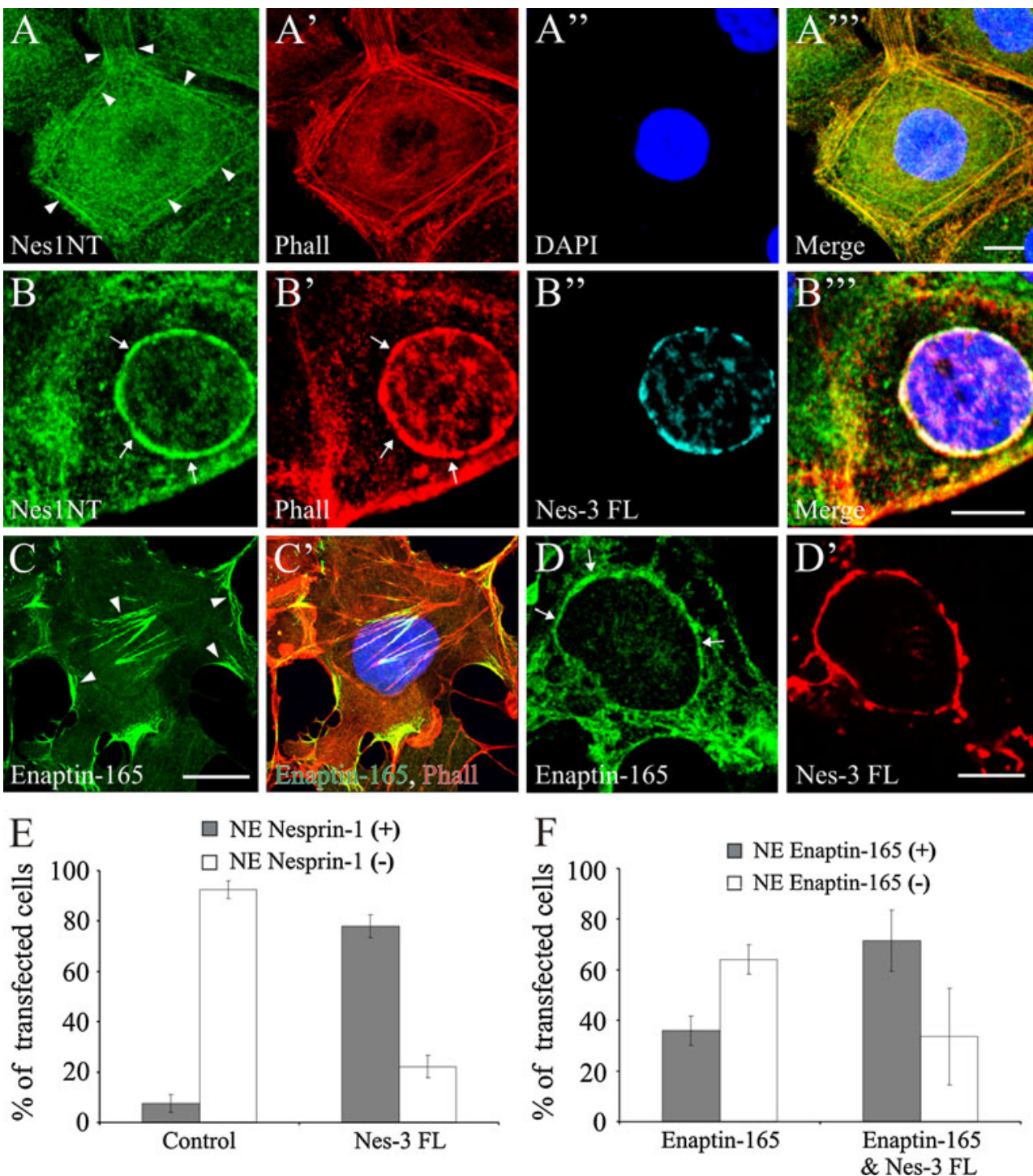

structural details) and purified His-tagged nesprin-2 ABD protein, with (Fig. 4b) or without (Fig. 4a) polymerized actin. In the absence of F-actin, GST-nes-3 SR1,2,3 precipitated specifically the nes-2 ABD protein (Fig. 4a, samples 2), whereas the latter remained in the supernatant (S) when GST was employed (Fig. 4a, samples 1). Therefore, GST-nes-3 SR1,2,3 binds directly to nes-2 ABD. In the presence of polymerized actin, the nes- 2 ABD co-sedimented with filamentous actin (P). Nevertheless, these complexes still remained largely absent from the pull-down (PD) fraction when GST alone was used (Fig. 4b, samples 3). When GST-nes-3 SR1,2,3 was used instead in the pull-down, both F-actin and nes-2 ABD proteins were found attached to the beads (Fig. 4b, samples 4). This data collectively suggests that the nesprin-2 ABD interaction with nesprin-3 and F-actin is not mutually exclusive.
The molecular mechanisms by which plectin and nesprin-1/-2 ABDs associate to nesprin-3 are not identical

To test which nesprin-3 domain interacted with the nesprin-1 and -2 ABDs, we performed GST pull-down assays. We engineered and purified three different GST fusions of the nesprin-3 $\alpha \mathrm{N}$-terminal spectrin repeats (SR), namely nes-3 SR1, SR1,2, and SR1,2,3 (see Fig. S1 for details). Nes-3 SR1 encompasses the first SR alone, whereas nes-3 SR1,2 and nes-3 SR1,2,3 contain the first two and three SRs of nesprin- $3 \alpha$ FL, respectively. Nes-3 SR1 corresponds to the major high affinity plectin ABD binding site of nesprin-3 and therefore was conceived as a positive control. Equal quantities of purified GST fusions including GST alone (negative control) were incubated with $\operatorname{COS} 7$ lysates that overexpressed the respective 


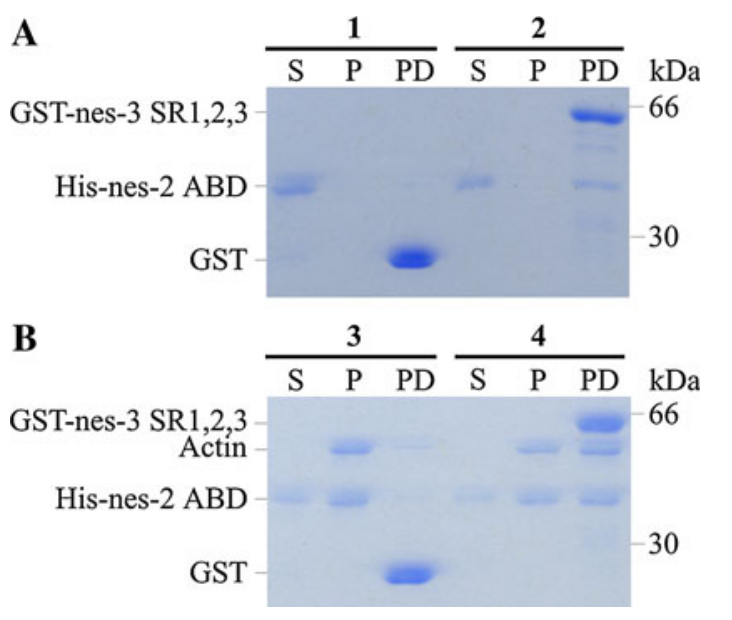

Fig. 4 a, b The nesprin-2 ABD associates to nesprin-3 in the presence of F-actin in vitro. Recombinantly expressed and purified His-nes-2 ABD protein was incubated either with GST (samples 1,3) or GST-nes-3 SR1,2,3 (samples 2,4) coupled Sepharose-beads in the absence (a) or presence of F-actin (b). Samples were then subjected to low speed centrifugation. The resulting supernatants from each experiment were ultra-centrifuged (see "Materials and methods" for more detail). The supernatants $(S)$, pellets $(P)$ from the ultracentrifugation, and the pellets from the GST pull-down assays $(P D$; first centrifugation step) were analyzed by SDS-PAGE and stained with Coomassie Blue. Note that the presence of filamentous actin does not preclude the nesprin-2 ABD from binding the nes-3 SR1,2,3 recombinant protein (samples 4 )

GFP-tagged ABD segments of plectin 1c, nesprin-1, and nesprin-2. The use of equal amounts of recombinant protein and the composition of the precipitated samples was investigated by immunoblotting. In contrast to GST alone, all the nesprin- 3 fusions exhibited binding to the ABDs tested. Nevertheless, and quite surprisingly, our data revealed distinct differences in the GST-fusion protein binding affinities to ABDs of nesprin-1, nesprin-2, and plectin. Both nesprin-1 and nesprin-2 ABDs behaved similarly and exhibited strong interactions with nes-3 SR1,2 and nes-3 SR1,2,3 fusions. However, only weak nesprin-1 and nesprin-2 ABD associations were evident with the shorter nesprin-3 SR1 construct (Fig. 5a). In contrast, the plectin $\mathrm{ABD}$ was efficiently precipitated by all three GST-nesprin-3 SR fusions that contained the SR1 segment (Fig. 5a). Overall, our data indicated that, while SR1 alone was sufficient to interact effectively with plectin, longer nesprin-3 fusions encompassing both SR1 and SR2 were required to mediate efficient nesprin-1/-2 ABD binding. Attempts to examine the binding of SR2 alone to the nesprin-1/-2 ABDs were not conclusive and hampered by the unstable nature of the recombinant protein (data not shown). Consistent with the above data, anti-myc immunoprecipitation of transiently expressed myc-tagged nes-3 FL from $\mathrm{HaCaT}$ cell homogenates revealed both plectin and nesprin-2 giant in the nesprin-3 immunoprecipitates (Fig. 5b).

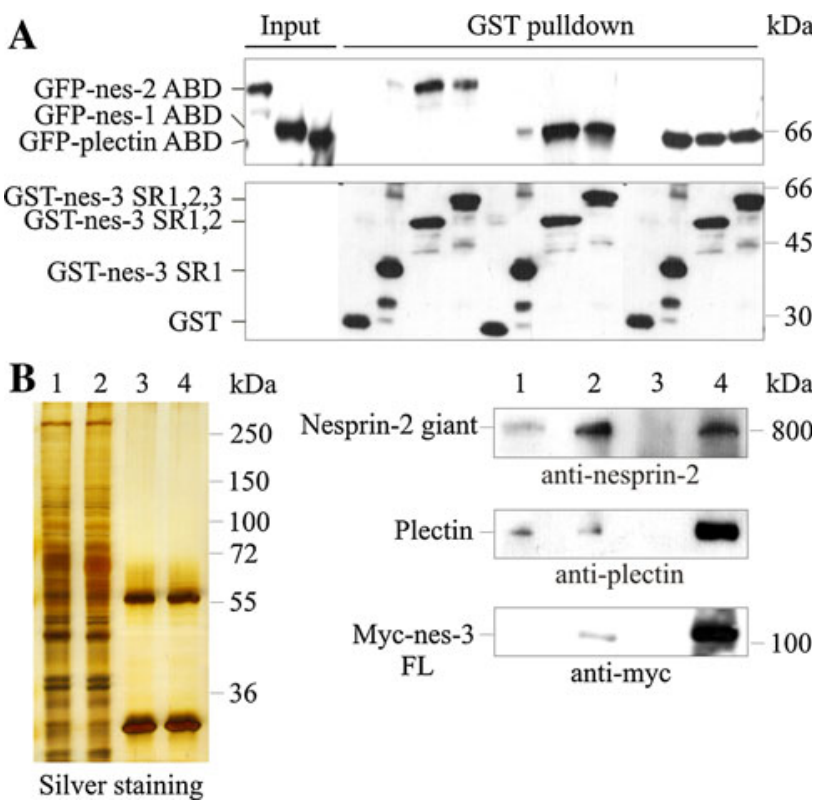

Fig. 5 a, b Nesprin-3 interacts in vitro and in vivo with plectin and the nesprin-1/-2 ABDs. a COS7 cells were transiently transfected with GFP-tagged nesprin-1 (nes-1), nesprin-2 (nes-2), or plectin ABDs and lysed in RIPA-buffer. Equal amounts of each lysate were incubated with either immobilized GST-fused nesprin-3 (nes-3) SR1, $S R 1,2, S R 1,2,3$, or $G S T$ alone (negative control). Specifically bound proteins were analyzed by SDS-PAGE and subjected to western blot analysis using either GFP (top panel) or GST specific antibodies (bottom panel). b Nesprin-2 giant co-immunoprecipitates with nesprin-3 and plectin. COS7 cells were transiently transfected with either empty myc-tag expression vector or myc-tagged nesprin-3 (nes3) FL. Cells were lysed in RIPA-buffer and subjected to anti-myc immunoprecipitation $(I P)$. Total cell lysates and myc-IPs were separated by SDS-PAGE and either stained directly with silver (left panel), or processed further for immunoblotting (right panel), using specific nesprin-2 (nes $2 C T$ ), plectin and myc antibodies. Gel loading information: lane 1 total COS7 cell lysate expressing empty myc-tag vector; lane 2 total COS7 cell homogenate expressing myc-tagged nesprin-3 (nes-3) FL; lane 3 myc only immunoprecipitate; lane 4 myc-nesprin-3 FL immunoprecipitates. Silver staining is used as a control to demonstrate equal cell lysate loading and IP specificity

Although we were unsuccessful in further narrowing the respective binding domains, our data suggested the testable possibility that nesprin-3 may be able to simultaneously bind to plectin and to either nesprin-1 or nesprin-2 as a direct consequence of harboring differential binding sites to these molecules.

Nesprin-3 does simultaneously recruit both the nesprin-2 ABD and plectin to the NE

To investigate the existence of tripartite nesprin-2/nesprin3/plectin interactions, we ectopically expressed HA-tagged nesprin-3 FL and determined if it could recruit endogenous plectin and overexpressed GFP-tagged nesprin-2 ABD (nes-2 ABD) in COS7 cells. Consistent with previous data [26], plectin immunofluorescence staining was primarily 
found throughout the cytoplasm in untransfected cells (Fig. 6a; Fig. S4B ${ }^{\prime \prime}$ ), with only $13 \%$ of cells exhibiting faint NE plectin staining (Fig. 6d). In sharp contrast, the overwhelming majority of cells ( $98 \%$, Fig. 6d) exhibited pronounced plectin NE rim staining when nes-3 FL was expressed (Fig. 6b-b" ${ }^{\prime \prime}$, d). Interestingly, in nes-3 FL and nesprin-2 ABD co-transfected cells, both the ABD-fusion and plectin were efficiently recruited to the NE (Fig. $\left.6 c-c^{\prime \prime \prime}\right)$.
These experiments show that the nesprin-3 binding to plectin and the nesprin-2 ABD is not mutually exclusive.

Next, we explored whether the nesprin-3 mediated redistribution of the nesprin-2 ABD requires plectin. To address this caveat, we examined the subcellular ABD pattern in control (Fig. 6f) and plectin siRNA treated (Fig. $6 \mathrm{~g}-\mathrm{g}^{\prime \prime}$ ) COS7 cells. The efficacy of our silencing strategy was evaluated in anti-plectin immunoblot
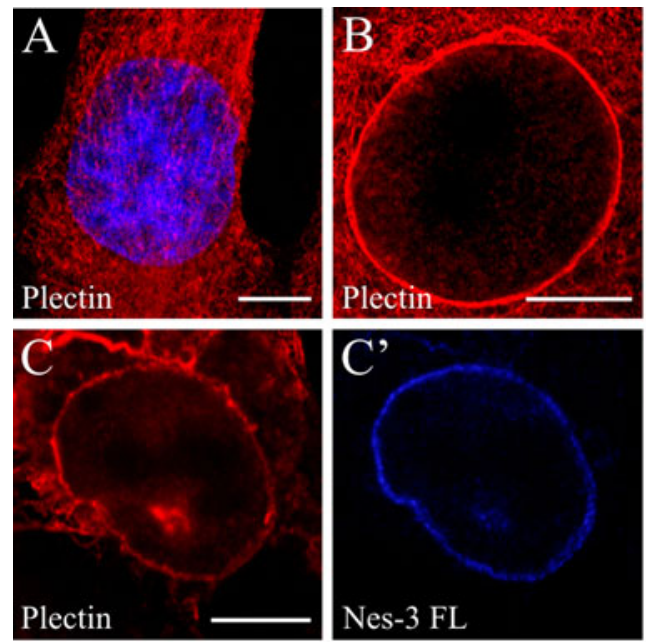

D
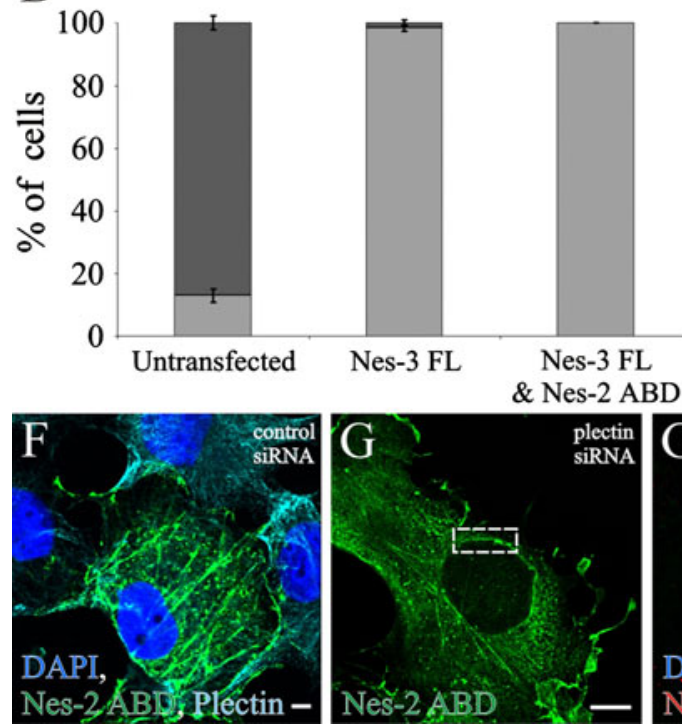

Fig. 6 a-g Nesprin-3 simultaneously recruits both plectin and the nesprin-2 ABD to the NE. a COS7 cells were stained for endogenous plectin (rabbit $\mathrm{Ab}$ ). Note the absence of plectin enrichment at the $\mathrm{NE}$ and the profound filamentous cytoplasmic plectin staining. $\mathbf{b}-\mathbf{b}^{\prime \prime}$ In HA-tagged nesprin-3 (nes-3) FL transfected COS7 cells, plectin was recruited to the NE. $\mathbf{c}-\mathbf{c}^{\prime \prime \prime}$ Both plectin and GFP-nesprin-2 (nes-2) ABD accumulated at the NE in GFP-nes-2 ABD/HA-nes-3 FL cotransfected cells. d Histogram representing the statistical evaluation of the NE plectin localization profiles in untransfected, in HA-nes-3 FL transfected and in GFP-nes-2 ABD/HA-nes-3 FL co-transfected COS7 cells. Note that nes-2 ABD recruitment by nesprin-3 does not have consequences on the plectin accumulation at the NE. Scale bars
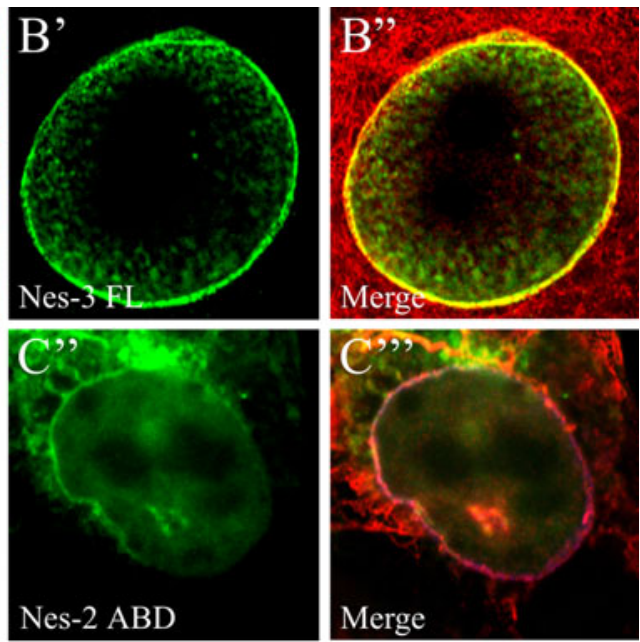

E
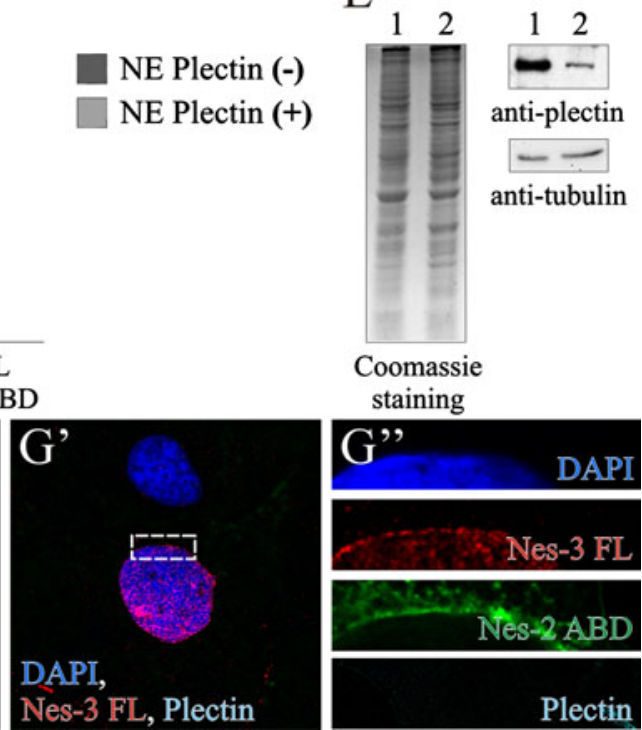

$10 \mu \mathrm{m}$. e Control (1) and plectin (2) siRNA treated COS7 lysates were analyzed by SDS-PAGE and either stained directly with Coomassie Blue (left panel; to demonstrate equal loading) or subjected to immunoblotting (right panels), using specific tubulin and plectin (to demonstrate silencing efficacy) antibodies. f Control siRNA treated COS7 cells, transiently expressing GFP-nes-2 ABD, exhibit profound nes-2 ABD filaments in the cytoplasm and plectin staining $\left(A b C\right.$-20). Scale bar $5 \mu \mathrm{m}$. $\mathbf{g}-\mathbf{g}^{\prime \prime}$ Plectin siRNA treated COS7 cells that transiently co-express nes-3 FL/nes-2 ABD constructs, lack endogenous plectin staining and possess prominent NEassociated ABD structures. Insets in $\mathbf{g}^{\prime \prime}$, are higher magnifications of dotted boxed areas in $\mathbf{g}$ and $\mathbf{g}^{\prime}$. Scale bar $10 \mu \mathrm{m}$ 
experiments, which demonstrated a significant plectin downregulation (Fig. 6e). In control silenced cells, both nesprin-2 ABD and plectin staining revealed pronounced cytoplasmic filamentous structures (Fig. 6f) with no obvious NE localization, as in untransfected controls (Fig. 6a). When plectin is downregulated, and nesprin-3 FL ectopically expressed (Fig. $6 \mathrm{~g}^{\prime}$ ), the nesprin-2 ABD again became prevalent at the NE (Fig. 6g), although plectin was largely undetectable (Fig. $6 \mathrm{~g}^{\prime \prime}$ ). Our data suggest that the nesprin-3 interaction with nesprin-2 is plectin independent.

Nesprin-2 and nesprin-3 associations control the nuclear morphology of $\mathrm{HaCaT}$ cells

Our current findings suggest that both $\mathrm{N}$ - and C-termini of nesprin-1 and -2 giant are integrated at the ONM. While the giant isoform $\mathrm{N}$-termini are attached indirectly via nesprin-3, their C-termini are directly embedded at the NE through the KASH/SUN-domain molecular bridge. As a consequence of these novel interactions, our data imply that nesprin-1 and - 2 giant isoforms may form a lattice that lies juxtaposed to the NE surface. To validate this novel nesprin arrangement, we used dominant negative approaches, that aimed to sever either the $\mathrm{N}$ - or C-terminal nesprin-2 giant "NE attachment sites". To saturate endogenous nesprin-2 giant N-terminal binding to nesprin3, we overexpressed GFP-tagged nesprin-2 ABD in HaCaT cells and examined its impact on nuclear shape by calculating the mid-plane cross-sectional nuclear area. Compared to untransfected HaCaT cells (Fig. 7a), transfected cells displayed increased nuclear areas (Fig. 7b, b'). A statistical evaluation revealed that the mean nuclear area values of GFP-nesprin-2 ABD overexpressing cells were 1.43-fold $\left(P<5 \times 10^{-14}\right)$ larger than those of untransfected cells (Fig. 7f). Similar nuclear area expansion effects (Fig. 7c, $\mathrm{c}^{\prime}, \mathrm{f}$ ) were obtained when we interrupted nesprin C-terminal domain anchorage by overexpressing the nesprin-2 KASH-domain (1.40-fold expansion, $P<5 \times 10^{-15}$ ). Taken together, these data indicated important roles for both the ABD and the KASH-domain in nuclear area maintenance. To further support our novel nesprin arrangement model, we decided to overexpress a GFP-tagged nesprin-2 mini construct (Fig. S1, [24]) and assess its effects on nuclear area. Nesprin-2 mini contains the $\mathrm{ABD}$ and the KASH-domain, which are required to bind nesprin-3 and SUN proteins, respectively. In contrast to nesprin-2 giant, however, nesprin-2 mini lacks the majority of the centrally located SRs. Therefore, our assumption was that nesprin-2 mini overexpression will function as a short molecular belt, which will tighten the nuclear perimeter and lead consequently to nuclear shrinkage. Consistent with this, the mean nuclear area values of GFP-nesprin-2 mini overexpressing cells were reduced to 0.87 -fold $(P<0.005)$ when compared to untransfected HaCaTs (Fig. 7d, d', f). This shows that the number of SRs in the massive nesprin-2 isoforms plays an important role in determining nuclear shape. Even more intriguing was our finding that the co-expression of GFPnesprin-2 mini and HA-tagged nes-3 FL resulted in a more significant $\left(0.77\right.$-fold, $\left.P<5 \times 10^{-9}\right)$ reduction of the nuclear area when compared to untransfected cells (Fig. 7e-e ${ }^{\prime \prime}, \mathrm{f}$ ). To exclude nesprin-2 SR interactions with nesprin-3, we overexpressed GFP-tagged nesprin-2 SRKASH (see Fig. S1 for construct details) alone or nesprin2 SR-KASH and nes-3 FL simultaneously. In brief, nesprin-2 SR-KASH encompasses the last four SR-domains and the entire KASH-domain of the nesprin-2 giant molecule. The nuclei of HaCaT cells expressing nesprin-2 SR-KASH or nesprin-2 SR-KASH and nes-3 FL were 1.29- or 1.30-fold larger than that of untransfected control cells, respectively (Fig. 7f). Consequently, the effects of nesprin-2 mini on nuclear shape are not the result of SR interactions.

Since nuclear and cell size are tightly coupled and regulated $[32,53]$ (Fig. S5A-A'), we next examined whether nesprin-2-derived domain overexpression also modulates the shape of transfected cells. Cell morphologies were visualized by phalloidin counter-staining (Fig. S5). Quantitative analysis indicated indeed significant cell shape changes upon nesprin-2 transgene expression (Fig. 7g; Fig. S5). Specifically, the area of control HaCaT cells $\left(1,033.7 \pm 370 \mu^{2}\right)$ became enlarged upon nesprin$2 \operatorname{ABD}\left(1,119.3 \pm 320 \mu^{2}\right)$ or KASH-domain $(1,210 \pm$ $332 \mu \mathrm{m}^{2}$ ) overexpression, while nesprin-2 mini positive cells exhibited smaller cell areas $\left(787 \pm 235 \mu^{2}\right)$ (Fig. 7g). These data indicate that nesprin-2-mediated nuclear shape changes are accompanied by analogous cellular changes, which is in agreement with previously published work [32]. Interestingly, however, while cell spreading was modulated, the expression levels of key cytoskeleton proteins, cell-substratum (vinculin), and cellcell (E-cadherin; data not shown) adhesion molecules remained unchanged (Fig. 7h). In summary, our data identify the nesprin-2 giant N-terminus and in particular the $\mathrm{ABD}$ as a pivotal structure that modulates both nuclear and cellular architecture.

Nesprin-2 mini and cytoskeleton-mediated tensions regulate nuclear size

Intrigued by our aforementioned findings, we next examined in more detail the role of the cytoskeleton on determining nuclear shape by chemically compromising cytoskeleton organization in transiently transfected nesprin-2 mini HaCaT cells. In untreated cells nesprin-2 mini expression induced nuclear shrinkage while remodeling 

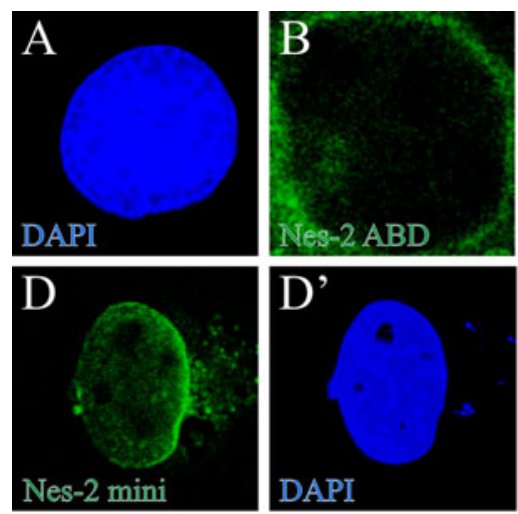

$\mathrm{F}$

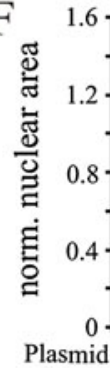

asmid:

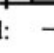

ABD
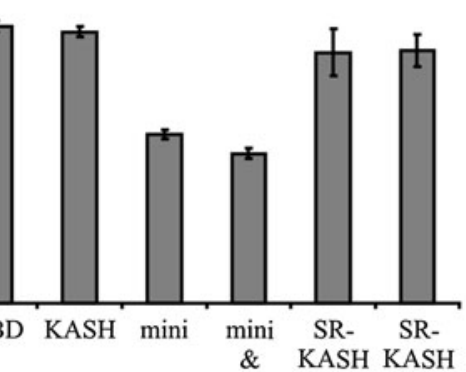

Nes-3 FL

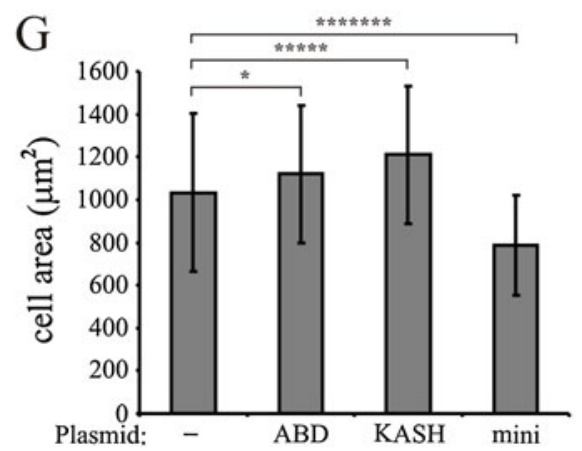

Fig. 7 a-h Nesprin-2 and neprin-3 associations synergistically control nuclear morphology. a The nucleus of an untransfected HaCaT cell stained with DAPI is shown. HaCaT cells expressing transiently GFP-nes-2 ABD (b-b') or GFP-nes-2 KASH (c, $\left.\mathbf{c}^{\prime}\right)$, display increased nuclear areas. In contrast, the nuclei of GFP-nes-2 mini transfected (d, $\mathbf{d}^{\prime}$ ) or GFP-Nes-2-mini/HA-Nes-3 FL co-transfected $\left(\mathbf{e}-\mathbf{e}^{\prime \prime}\right)$ HaCaT cells are smaller than those of untransfected cells. DNA was stained with DAPI. Scale bars $10 \mu \mathrm{m}$. f Histogram indicates the normalized nuclear areas of untransfected and GFP-nes2 ABD, GFP-nes-2 KASH, GFP-nes-2 mini, GFP-nes-2 mini/HA-nes3 FL, GFP-SR-KASH, GFP-SR-KASH/HA-nes-3 FL expressing $\mathrm{HaCaT}$ cells. The mean nuclear area of untransfected HaCaTs was normalized to 1 . The normalized mean nuclear area was $1.43 \pm 0.03$

effects on F-actin and microtubule structures around the NE were not discernible when compared to untransfected cells (Fig. 8a-a $\mathrm{a}^{\prime \prime \prime}$ ). Upon treatment with latrunculin B (LatB), which inhibits microfilament polymerization we noticed, as anticipated, reduced phalloidin staining and major effects on nuclear shape. Compared to untreated cells, nuclei were highly misshapen and exhibited smaller

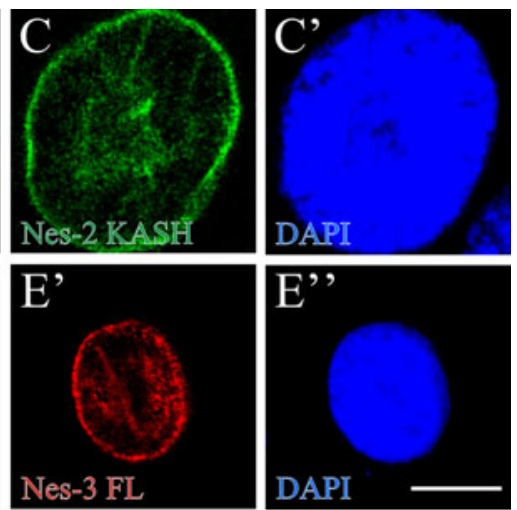

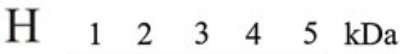

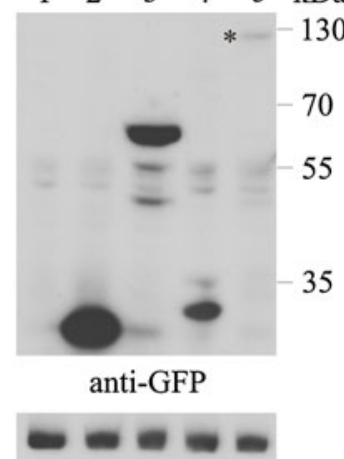

anti-vinculin

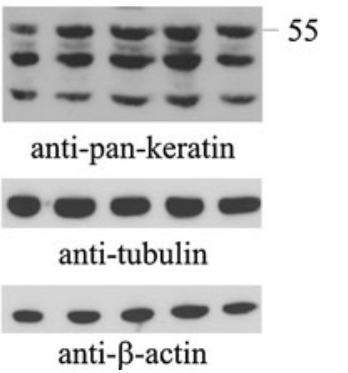

for GFP-nes-2 ABD, $1.40 \pm 0.03$ for GFP-nes-2 KASH, $0.87 \pm 0.02$ for GFP-nes-2 mini, $0.77 \pm 0.03$ for GFP-nes-2 mini/HA-nes-3 FL, $1.29 \pm 0.1$ for GFP-SR-KASH, and $1.30 \pm 0.08$ for GFP-SR-KASH/ HA-nes-3 FL cotransfected HaCaT cells. G Histogram indicating that, GFP-nes-2 ABD, nes-2 KASH overexpression leads to cellular enlargement while nes-2 mini yields smaller cells; * indicates statistically significant differences obtained by Student's $t$ test (200 cells counted for each experiment). Values in (f, g) are the mean \pm SD. h Western blot analysis of equal amounts of untransfected (1), GFP (2), GFP-nes-2 ABD (3), GFP-nes-2 KASH (4), and GFP-nes-2 mini (5) HaCaT lysates shows that cytoskeletal and vinculin protein expression is not altered; * in top panel indicates GFP-nes-2 mini

nuclear areas, including nes-2 mini positive cells (Fig. 8b, $\mathrm{b}^{\prime}$, e). In stark contrast, colchicine (Colch) induced microtubule depolymerization led to nuclear expansion, which was particularly pronounced in untransfected cells (Fig. 8c, $\mathrm{c}^{\prime}$, e). Nuclei, however, were not as irregular as under the F-actin depolymerizing conditions. Strikingly, the combined application of the cytoskeleton drugs led to 
similar nuclear shrinkage effects (Fig. $8 \mathrm{~d}-\mathrm{d}^{\prime \prime \prime}$, e) as in latrunculin B treated cells. These results highlight that the cytoskeleton is a key regulator of nuclear shape, including cells expressing nes- 2 mini, considering that all the outlined effects were statistically significant when evaluated relative to the corresponding untreated control samples $(P<0.05)$. While the effects of nes- 2 mini on nuclear size were modulated by cytoskeleton drugs, its expression still displayed significant constriction actions when compared to untransfected nuclei (Fig. 8e).

\section{Discussion}

Nesprins control fundamental aspects of the cell such as architecture, asymmetry, stiffness and even signaling [12,
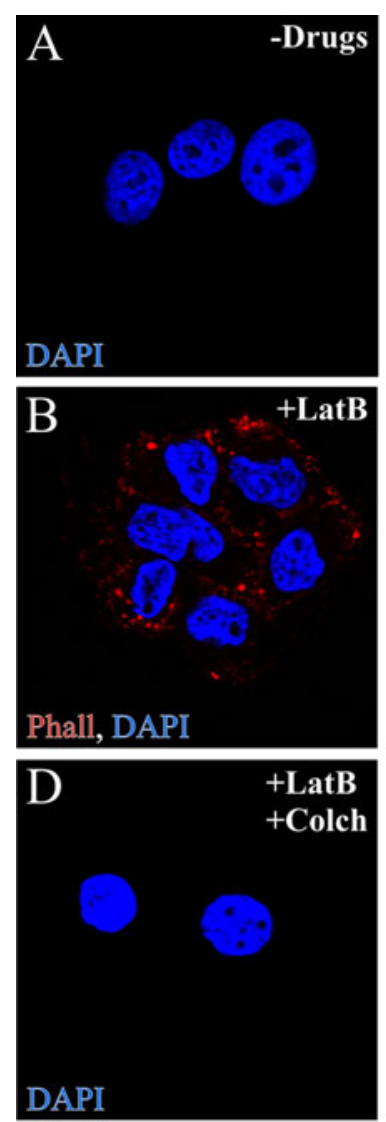
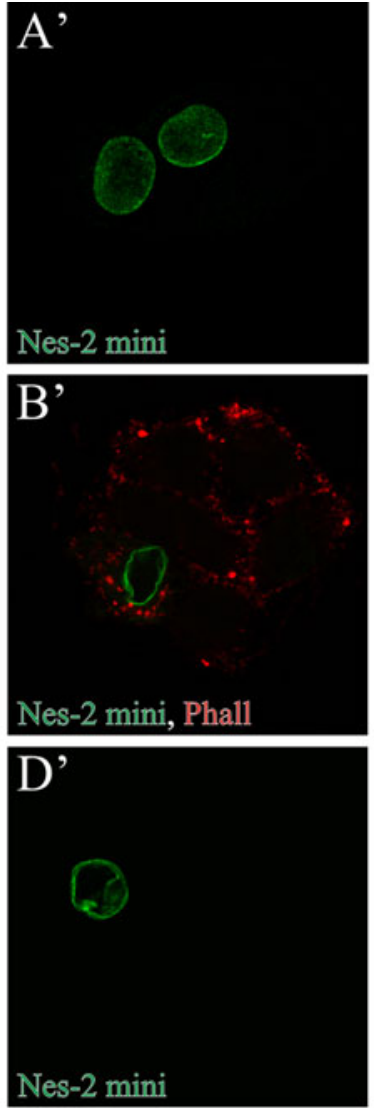
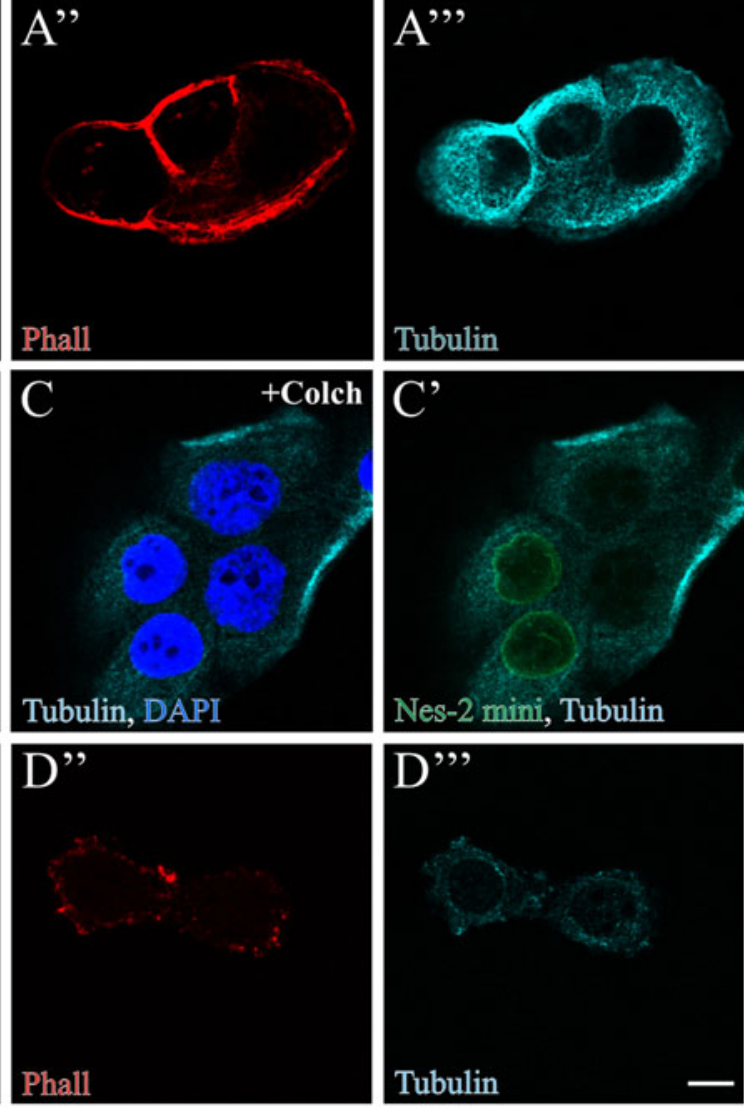

E

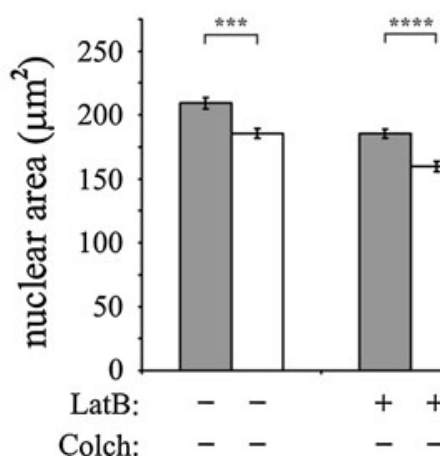

Fig. 8 a-e Nes-2 mini and the cytoskeleton regulate nuclear shape. a-a $\mathbf{a}^{\prime \prime \prime}$ Representative GFP-nes-2 mini induced nuclear and cellular shrinkage effects in untreated HaCaTs. Note that effects of nes- 2 mini expression on F-actin (Phall) and microtubule structures are not obvious. b, $\mathbf{b}^{\prime}$ Latrunculin B (LatB) induced F-actin depolymerization yields smaller and highly irregular nuclei. c, $\mathbf{c}^{\prime}$ Colchicine (Colch) induced microtubule depolymerization enlarges nuclei. $\mathbf{d}, \mathbf{d}^{\prime \prime \prime}$ Latrunculin B and colchicine co-treated cells harbor smaller nuclei.

Note that under all examined conditions that nes- 2 mini expressing nuclei are more compact than control nuclei. DAPI was used to stain nuclei. Scale bar $10 \mu \mathrm{m}$. e Histogram representing the nuclear areas of untreated, latrunculin B, colchicine treated, and co-treated, control (untransfected) and nes-2 mini expressing HaCaT cells. Values are the mean $\pm \mathrm{SEM}$; * indicates statistically significant differences obtained by Student's $t$ test (100 nuclei counted for each condition) 
$22,38,54]$. How these molecules master all these multiple functions is poorly understood. In this paper, we highlight the structural nesprin-1/-2 giant molecule features by providing novel insights into their associations with nesprin-3 and by discussing their possible arrangement at the NE.

The first nesprin- $3 \alpha$ spectrin repeat (SR1; plakin-binding domain) was already known to associate with the ABDs of the cytoskeletal linker proteins plectin and MACF1 [26]. Here, we further expand the nesprin-3 complex repertoire by revealing novel associations with the nesprin-1/-2 ABDs both in vitro and in vivo. Our studies, however, identify differences between the implicated domains of nesprin-3. More specifically, our data show that, in contrast to plectin, nesprin1/-2 ABDs interact weakly with nesprin-3 SR1. Only the use of larger nesprin-3 fusions, that contain in addition the second spectrin repeat (SR2), increases its affinity to the ABDs. This result suggests that either nesprin-3 contains multiple nesprin-1/-2 ABD binding sites or that SR2 allows proper SR1 folding and thereby increases its affinity to nesprin-1/-2. We cannot exclude the latter, but do favor the former scenario. This interpretation is based on the presence of both plectin and nesprin-2 giant in the nesprin-3 immunoprecipitates. Furthermore, the nesprin-3-mediated recruitment of nesprin$2 \mathrm{ABD}$ coincided with the repositioning of plectin to the $\mathrm{NE}$ in transfected cells. Thus, tripartite nesprin-2/nesprin-3 and plectin complexes can be formed. Importantly, we also showed that the capacity of nesprin-3 to associate with the nesprin-2 ABD does not compromise their ability to recruit F-actin. Collectively, this indicates that nesprin-3 may integrate not only intermediate filaments but also F-actin (via nesprins-1/-2 giant) at the nuclear surface.

While we do not underestimate the biological significance of versatile cytoskeletal nesprin-3 attachment sites, we are particularly intrigued by the physical consequences of nesprin-3 binding on the arrangement of the nesprin-1/-2 giant molecules at the NE. By capturing the N-terminal ends of these massive proteins, nesprin-3 would in principle align them along the ONM. Such a model is not consistent with current schematics depicting the giant nesprin molecules as radiating filaments projecting into the cytoplasm. However, several lines of evidence support the novel model that we propose here. Firstly, massive proteins usually have differential immunofluorescence patterns of their $\mathrm{N}$ - and C-terminal epitopes. For instance, the nebulin C-terminus (600-900 kDa) localizes at Z-discs, while its $\mathrm{N}$-terminus is distinctively positioned towards the sarcomere centre [55]. Importantly, however, the nesprin-2 giant staining pattern using antibodies directed against the $\mathrm{ABD}$ and its C-terminal domain is largely identical [49]. This finding suggests that the nesprin-2 giant $\mathrm{N}$-terminus is positioned near the nuclear membrane and close to the C-terminus of another nesprin-2 molecule. Secondly, most spectrin-containing molecules are aligned along membrane surfaces and do not protrude into the cytoplasm. In red blood cells, spectrin lines the intracellular side of the plasma membrane forming a filamentous network. This two-dimensional spectrin meshwork provides a scaffold for a variety of proteins and plays important roles in cell shape maintenance [56]. Studies on isolated membranes and phospholipid vesicles also indicate bilayer lamination by spectrin dimers [57]. Dystrophin, another spectrin-containing protein is also suspected to align the plasma membrane and to create a link between the intracellular cytoskeleton and the extracellular matrix via the dystrophin-associated protein complex in muscle [58-61]. Finally, the strongest evidence that giant nesprin molecules line the outer NE surface comes from our studies that reveal important roles in nuclear shape control. The interference with the nesprin-2 giant molecule ends triggers nuclear enlargement, whereas the overexpression of nesprin-2 mini leads to nuclear compaction. Our hypothesis is that nesprin-2 mini dislodges endogenous giant isoforms from the NE and that its presence tightens (as a consequence of its drastically shorter rod domain) the nesprin-1/2/-3 "belt" that would normally encapsulate the NE. Consistent with this scenario is that primary cells obtained from nesprin-2 giant knockout mice show increased nuclear sizes and severely misshapen nuclei [32]. Intriguingly, the expression of nesprin-2 giant isoforms, that exclusively lack the bulk of the ABD (Nesprin-2 $\triangle \mathrm{ABD}$ ) and which still contain the remainder of the molecule, rescue the NE deformation phenotype, but cannot reverse the increased nuclear area defects of nesprin-2 giant null cells. This result therefore in particular underlines the role of the ABD in nuclear shape regulation.

In conclusion, apart from its involvement in actinmediated nuclear movement [37], the nesprin-2 ABD harbors additional functions in maintaining nuclear shape. Our assumption is that this is facilitated by interactions with nesprin-3 and possibly additional unknown associations with other NE constituents. Clearly, major important co-players are the cytoskeleton and the motor protein apparatus, elements to which nesprins have tight associations and which are concomitantly well-known nuclear shape regulators $[36,62]$. Although nesprin-2 mini lacks a major microtubule-associating domain (i.e. kinesin light chain 1 binding site [22]), and while its expression does not yield obvious peri-nuclear cytoskeleton rearrangements in keratinocytes (contrary to what has been reported in COS7 cells [24]), our data show that nesprin-2 mini positive nuclei are integrated and subject to opposing cytoskeletonassociated forces. While chemically induced F-actin depolymerization further shrinks nuclei, microtubule disruption leads to nuclear expansion. These findings are consistent with current reports $[36,63]$ that indicate that nuclei are under a pre-stressed state, whereby actomyosin 
complexes apply outward pulling forces while microtubulebased structures exert compressing actions. Altogether, this illustrates that nuclear shape is the net outcome of complex intrinsic and extrinsic interactions that include integral NE constituents themselves (e.g., nesprins), as well as structures on the opposing sites of the nuclear membranes, such as the nuclear lamina, the genome, and the cytoskeleton [36, 62-65].

Considering the pronounced SUN and KASH-domain protein oligomerization properties, one can assume that such associations may jointly organize these molecules into larger complexes. In fact, nesprin molecules themselves may contribute to an expansion and the formation of even larger macromolecular complexes. Nesprin-1 was shown to oligomerize with itself, through its $\mathrm{N}$ - and C-terminal spectrin repeats $[66,67]$. Also, nesprin-3 oligomerizes with itself, through its N-terminal spectrin repeats [28]. Furthermore, SUN proteins form immobile macromolecular and promiscuous assemblies $[20,68]$ at the NE that may provide multiple docking sites for both $\mathrm{N}$ - and $\mathrm{C}$-terminal nesprin ends. As a consequence, nesprins in conjunction with the cytoskeleton may form a multifunctional 3-D meshwork of filaments that encapsulates the ONM.

In summary, we propose a new model whereby nesprin interchain associations favor the formation of a basket-like

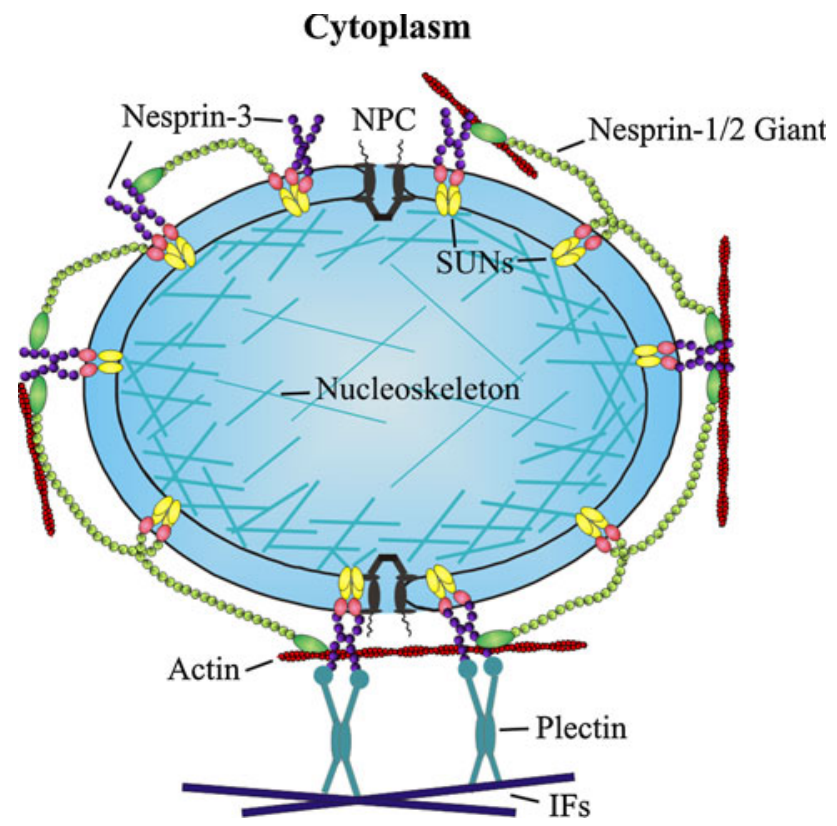

Fig. 9 Model illustrating nesprin-1/-2/-3 interchain interactions and links with the cytoskeleton at the nuclear surface. Nesprins-1/-2 giant isoforms are drawn to align the NE perimeter. Nesprin-1/-2 ABDs interact with F-actin and nesprin-3, the latter is shown to interact also with the cytolinker plectin. Thus, nesprin-cytoskeleton complexes form a 3-D filamentous network that covers the NE exterior, which functions as a molecular "belt" (see "Discussion" for details). SUNdomain proteins are depicted as dimers and tetramers. IFs intermediate filaments, NPC nuclear pore complex protein network that covers and supports the cytoplasmic NE surface (Fig. 9). Its existence may be pivotal not only to structure nuclei but also key in providing the necessary framework to effectively transduce forces to the nuclear surface in order to conduct nuclear movement and thus to establish cell polarity [22, 37, 69].

Acknowledgments We thank Drs Arto Maatta and Arnoud Sonnenberg for providing reagents and Drs. Patrick Hussey, Andrei Smertenko, and Roy Quinlan for valuable discussions. We are also grateful to Dr. Tim Hawkins for excellent advice on confocal microscopy. This work was supported by the Deutsche Forschungsgemeinschaft (DFG; KA 2778/1-1) and a Wellcome Trust "Value in People" award to I.K.. W.L., and S.T. were members of the NRW International Graduate School in Development and Disease (IGSDHD), Cologne.

\section{References}

1. Gerace L, Burke B (1988) Functional organization of the nuclear envelope. Annu Rev Cell Biol 4:335-374

2. Starr DA (2007) Communication between the cytoskeleton and the nuclear envelope to position the nucleus. Mol BioSyst 3:583-589

3. Wilson KL, Berk JM (2010) The nuclear envelope at a glance. J Cell Sci 123:1973-1978

4. Stuurman N, Heins S, Aebi U (1998) Nuclear lamins: their structure, assembly, and interactions. J Struct Biol 122:42-66

5. Burke B, Ellenberg J (2002) Remodelling the walls of the nucleus. Nat Rev Mol Cell Biol 3:487-497

6. Broers JL, Ramaekers FC, Bonne G, Yaou RB, Hutchison CJ (2006) Nuclear lamins: laminopathies and their role in premature ageing. Physiol Rev 86:967-1008

7. Mattout A, Dechat T, Adam SA, Goldman RD, Gruenbaum Y (2006) Nuclear lamins, diseases and aging. Curr Opin Cell Biol 18:335-341

8. Schneider M, Noegel AA, Karakesisoglou I (2008) KASHdomain proteins and the cytoskeletal landscapes of the nuclear envelope. Biochem Soc Trans 36:1368-1372

9. Xiong H, Rivero F, Euteneuer U, Mondal S, Mana-Capelli S, Larochelle D, Vogel A, Gassen B, Noegel AA (2008) Dictyostelium Sun-1 connects the centrosome to chromatin and ensures genome stability. Traffic 9:708-724

10. Schulz I, Baumann O, Samereier M, Zoglmeier C, Graf R (2009) Dictyostelium Sun1 is a dynamic membrane protein of both nuclear membranes and required for centrosomal association with clustered centromeres. Eur J Cell Biol 88:621-638

11. Graumann K, Evans DE (2010) Plant SUN domain proteins: components of putative plant LINC complexes? Plant Signal Behav 5:154-156

12. Starr DA, Fridolfsson HN (2010) Interactions between nuclei and the cytoskeleton are mediated by SUN-KASH nuclear-envelope bridges. Annu Rev Cell Dev Biol 26:421-444

13. Zhou K, Hanna-Rose W (2010) Movers and shakers or anchored: caenorhabditis elegans nuclei achieve it with KASH/SUN. Dev Dyn 239:1352-1364

14. Padmakumar VC, Libotte T, Lu W, Zaim H, Abraham S, Noegel AA, Gotzmann J, Foisner R, Karakesisoglou I (2005) The inner nuclear membrane protein Sun1 mediates the anchorage of Nesprin-2 to the nuclear envelope. J Cell Sci 118:3419-3430

15. Crisp M, Liu Q, Roux K, Rattner JB, Shanahan C, Burke B, Stahl PD, Hodzic D (2006) Coupling of the nucleus and cytoplasm: role of the LINC complex. J Cell Biol 172:41-53 
16. Haque F, Lloyd DJ, Smallwood DT, Dent CL, Shanahan CM, Fry AM, Trembath RC, Shackleton S (2006) SUN1 interacts with nuclear lamin A and cytoplasmic nesprins to provide a physical connection between the nuclear lamina and the cytoskeleton. Mol Cell Biol 26:3738-3751

17. Jaspersen SL, Martin AE, Glazko G, Giddings TH Jr, Morgan G, Mushegian A, Winey M (2006) The Sad1-UNC-84 homology domain in Mps3 interacts with Mps2 to connect the spindle pole body with the nuclear envelope. J Cell Biol 174:665-675

18. McGee MD, Rillo R, Anderson AS, Starr DA (2006) UNC-83 IS a KASH protein required for nuclear migration and is recruited to the outer nuclear membrane by a physical interaction with the SUN protein UNC-84. Mol Biol Cell 17:1790-1801

19. Kracklauer MP, Banks SM, Xie X, Wu Y, Fischer JA (2007) Drosophila klaroid encodes a SUN domain protein required for Klarsicht localization to the nuclear envelope and nuclear migration in the eye. Fly (Austin) 1:75-85

20. Lu W, Gotzmann J, Sironi L, Jaeger VM, Schneider M, Luke Y, Uhlen M, Szigyarto CA, Brachner A, Ellenberg J, Foisner R, Noegel AA, Karakesisoglou I (2008) Sun1 forms immobile macromolecular assemblies at the nuclear envelope. Biochim Biophys Acta 1783:2415-2426

21. Starr DA, Fischer JA (2005) KASH 'n Karry: the KASH domain family of cargo-specific cytoskeletal adaptor proteins. BioEssays 27:1136-1146

22. Schneider M, Lu W, Neumann S, Brachner A, Gotzmann J, Noegel AA, Karakesisoglou I (2011) Molecular mechanisms of centrosome and cytoskeleton anchorage at the nuclear envelope. Cell Mol Life Sci 68:1593-1610

23. Zhang Q, Skepper JN, Yang F, Davies JD, Hegyi L, Roberts RG, Weissberg PL, Ellis JA, Shanahan CM (2001) Nesprins: a novel family of spectrin-repeat-containing proteins that localize to the nuclear membrane in multiple tissues. J Cell Sci 114:4485-4498

24. Zhen YY, Libotte T, Munck M, Noegel AA, Korenbaum E (2002) NUANCE, a giant protein connecting the nucleus and actin cytoskeleton. J Cell Sci 115:3207-3222

25. Padmakumar VC, Abraham S, Braune S, Noegel AA, Tunggal B, Karakesisoglou I, Korenbaum E (2004) Enaptin, a giant actinbinding protein, is an element of the nuclear membrane and the actin cytoskeleton. Exp Cell Res 295:330-339

26. Wilhelmsen K, Litjens SH, Kuikman I, Tshimbalanga N, Janssen H, van den Bout I, Raymond K, Sonnenberg A (2005) Nesprin-3, a novel outer nuclear membrane protein, associates with the cytoskeletal linker protein plectin. J Cell Biol 171:799-810

27. Roux KJ, Crisp ML, Liu Q, Kim D, Kozlov S, Stewart CL, Burke B (2009) Nesprin 4 is an outer nuclear membrane protein that can induce kinesin-mediated cell polarization. Proc Natl Acad Sci USA 106:2194-2199

28. Ketema M, Wilhelmsen K, Kuikman I, Janssen H, Hodzic D, Sonnenberg A (2007) Requirements for the localization of nesprin-3 at the nuclear envelope and its interaction with plectin. J Cell Sci 120:3384-3394

29. Cottrell JR, Borok E, Horvath TL, Nedivi E (2004) CPG2: a brain- and synapse-specific protein that regulates the endocytosis of glutamate receptors. Neuron 44:677-690

30. Kobayashi Y, Katanosaka Y, Iwata Y, Matsuoka M, Shigekawa M, Wakabayashi S (2006) Identification and characterization of GSRP-56, a novel Golgi-localized spectrin repeat-containing protein. Exp Cell Res 312:3152-3164

31. Zhang X, Xu R, Zhu B, Yang X, Ding X, Duan S, Xu T, Zhuang Y, Han M (2007) Syne-1 and Syne-2 play crucial roles in myonuclear anchorage and motor neuron innervation. Development 134:901-908

32. Luke Y, Zaim H, Karakesisoglou I, Jaeger VM, Sellin L, Lu W, Schneider M, Neumann S, Beijer A, Munck M, Padmakumar VC, Gloy J, Walz G, Noegel AA (2008) Nesprin-2 Giant (NUANCE) maintains nuclear envelope architecture and composition in skin. J Cell Sci 121:1887-1898

33. Stewart-Hutchinson PJ, Hale CM, Wirtz D, Hodzic D (2008) Structural requirements for the assembly of LINC complexes and their function in cellular mechanical stiffness. Exp Cell Res 314:1892-1905

34. Dawe HR, Adams M, Wheway G, Szymanska K, Logan CV, Noegel AA, Gull K, Johnson CA (2009) Nesprin-2 interacts with meckelin and mediates ciliogenesis via remodelling of the actin cytoskeleton. J Cell Sci 122:2716-2726

35. Zhang X, Lei K, Yuan X, Wu X, Zhuang Y, Xu T, Xu R, Han M (2009) SUN1/2 and Syne/Nesprin-1/2 complexes connect centrosome to the nucleus during neurogenesis and neuronal migration in mice. Neuron 64:173-187

36. Chancellor TJ, Lee J, Thodeti CK, Lele T (2010) Actomyosin tension exerted on the nucleus through nesprin-1 connections influences endothelial cell adhesion, migration, and cyclic straininduced reorientation. Biophys J 99:115-123

37. Luxton GW, Gomes ER, Folker ES, Vintinner E, Gundersen GG (2010) Linear arrays of nuclear envelope proteins harness retrograde actin flow for nuclear movement. Science 329:956-959

38. Neumann S, Schneider M, Daugherty RL, Gottardi CJ, Eming SA, Beijer A, Noegel AA, Karakesisoglou I (2010) Nesprin-2 interacts with $\{$ alpha $\}$-catenin and regulates Wnt signaling at the nuclear envelope. J Biol Chem 285:34932-34938

39. Sjöblom T, Jones S, Wood LD, Parsons DW, Lin J, Barber TD, Mandelker D, Leary RJ, Ptak J, Silliman N, Szabo S, Buckhaults P, Farrell C, Meeh P, Markowitz SD, Willis J, Dawson D, Willson JK, Gazdar AF, Hartigan J, Wu L, Liu C, Parmigiani G, Park BH, Bachman KE, Papadopoulos N, Vogelstein B, Kinzler KW, Velculescu VE (2006) The consensus coding sequences of human breast and colorectal cancers. Science 314:268-274

40. Gros-Louis F, Dupre N, Dion P, Fox MA, Laurent S, Verreault S, Sanes JR, Bouchard JP, Rouleau GA (2007) Mutations in SYNE1 lead to a newly discovered form of autosomal recessive cerebellar ataxia. Nat Genet 39:80-85

41. Kandert S, Lüke Y, Kleinhenz T, Neumann S, Lu W, Jaeger VM, Munck M, Wehnert M, Müller CR, Zhou Z, Noegel AA, Dabauvalle MC, Karakesisoglou I (2007) Nesprin-2 giant safeguards nuclear envelope architecture in LMNA S143F progeria cells. Hum Mol Genet 16:2944-2959

42. Zhang Q, Bethmann C, Worth NF, Davies JD, Wasner C, Feuer A, Ragnauth CD, Yi Q, Mellad JA, Warren DT, Wheeler MA, Ellis JA, Skepper JN, Vorgerd M, Schlotter-Weigel B, Weissberg PL, Roberts RG, Wehnert M, Shanahan CM (2007) Nesprin-1 and -2 are involved in the pathogenesis of Emery Dreifuss muscular dystrophy and are critical for nuclear envelope integrity. Hum Mol Genet 16:2816-2833

43. Marmé A, Zimmermann HP, Moldenhauer G, Schorpp-Kistner M, Müller C, Keberlein O, Giersch A, Kretschmer J, Seib B, Spiess E, Hunziker A, Merchán F, Möller P, Hahn U, Kurek R, Marmé F, Bastert G, Wallwiener D, Ponstingl H (2008) Loss of Drop1 expression already at early tumor stages in a wide range of human carcinomas. Int J Cancer 123:2048-2056

44. Attali R, Warwar N, Israel A, Gurt I, McNally E, Puckelwartz M, Glick B, Nevo Y, Ben-Neriah Z, Melki J (2009) Mutation of SYNE-1, encoding an essential component of the nuclear lamina, is responsible for autosomal recessive arthrogryposis. Hum Mol Genet 18:3462-3469

45. Haque F, Mazzeo D, Patel JT, Smallwood DT, Ellis JA, Shanahan CM, Shackleton S (2010) Mammalian SUN protein interaction networks at the inner nuclear membrane and their role in laminopathy disease processes. J Biol Chem 285:3487-3498

46. Puckelwartz MJ, Kessler EJ, Kim G, Dewitt MM, Zhang Y, Earley JU, Depreux FF, Holaska J, Mewborn SK, Pytel P, 
McNally EM (2010) Nesprin-1 mutations in human and murine cardiomyopathy. J Mol Cell Cardiol 48:600-608

47. Puckelwartz MJ, Kessler E, Zhang Y, Hodzic D, Randles KN, Morris G, Earley JU, Hadhazy M, Holaska JM, Mewborn SK, Pytel P, McNally EM (2009) Disruption of nesprin-1 produces an Emery Dreifuss muscular dystrophy-like phenotype in mice. Hum Mol Genet 18:607-620

48. Zhang J, Felder A, Liu Y, Guo LT, Lange S, Dalton ND, Gu Y, Peterson KL, Mizisin AP, Shelton GD, Lieber RL, Chen J (2010) Nesprin 1 is critical for nuclear positioning and anchorage. Hum Mol Genet 19:329-341

49. Libotte T, Zaim H, Abraham S, Padmakumar VC, Schneider M, Lu W, Munck M, Hutchison C, Wehnert M, Fahrenkrog B, Sauder U, Aebi U, Noegel AA, Karakesisoglou I (2005) Lamin A/C Dependent Localization of Nesprin-2, a Giant Scaffolder at the Nuclear Envelope. Mol Biol Cell 16:3411-3424

50. Noegel AA, Blau-Wasser R, Sultana H, Muller R, Israel L, Schleicher M, Patel H, Weijer CJ (2004) The cyclase-associated protein CAP as regulator of cell polarity and cAMP signaling in Dictyostelium. Mol Biol Cell 15:934-945

51. Evan GI, Lewis GK, Ramsay G, Bishop JM (1985) Isolation of monoclonal antibodies specific for human c-myc proto-oncogene product. Mol Cell Biol 5:3610-3616

52. Towbin H, Staehelin T, Gordon J (1979) Electrophoretic transfer of proteins from polyacrylamide gels to nitrocellulose sheets: procedure and some applications. Proc Natl Acad Sci USA 76:4350-4354

53. Neumann FR, Nurse P (2007) Nuclear size control in fission yeast. J Cell Biol 179:593-600

54. Olins AL, Hoang TV, Zwerger M, Herrmann H, Zentgraf H, Noegel AA, Karakesisoglou I, Hodzic D, Olins DE (2009) The LINC-less granulocyte nucleus. Eur J Cell Biol 88:203-214

55. Wright J, Huang QQ, Wang K (1993) Nebulin is a full-length template of actin-filaments in the skeletal-muscle sarcomere-an immunoelectron microscopic study of its orientation and span with site-specific monoclonal-antibodies. J Muscle Res Cell Motil 14:476-483

56. Chakrabarti A, Kelkar DA, Chattopadhyay A (2006) Spectrin organization and dynamics: new insights. Biosci Rep 26:369-386

57. Ray S, Chakrabarti A (2004) Membrane interaction of erythroid spectrin: surface-density-dependent high-affinity binding to phosphatidylethanolamine. Mol Membr Biol 21:93-100
58. Blake DJ, Weir A, Newey SE, Davies KE (2002) Function and genetics of dystrophin and dystrophin-related proteins in muscle. Physiol Rev 82:291-329

59. Nowak KJ, Davies KE (2004) Duchenne muscular dystrophy and dystrophin: pathogenesis and opportunities for treatment. EMBO Rep 5:872-876

60. Zhou GQ, Xie HQ, Zhang SZ, Yang ZM (2006) Current understanding of dystrophin-related muscular dystrophy and therapeutic challenges ahead. Chin Med J 119:1381-1391

61. Le Rumeur E, Winder SJ, Hubert JF (2010) Dystrophin: more than just the sum of its parts. Biochim Biophys Acta 1804:1713-1722

62. Khatau SB, Hale CM, Stewart-Hutchinson PJ, Patel MS, Stewart CL, Searson PC, Hodzic D, Wirtz D (2009) A perinuclear actin cap regulates nuclear shape. Proc Natl Acad Sci USA 106:19017-19022

63. Mazumder A, Shivashankar GV (2010) Emergence of a prestressed eukaryotic nucleus during cellular differentiation and development. J R Soc Interface 7:321-330

64. Polychronidou M, Grobhans J (2011) Determining nuclear shape: the role of farnesylated nuclear membrane proteins. Nucleus 2:17-23

65. Webster M, Witkin KL, Cohen-Fix O (2009) Sizing up the nucleus: nuclear shape, size and nuclear-envelope assembly. J Cell Sci 122:1477-1486

66. Taranum S, Sur I, Müller R, Lu W, Rashmi RN, Munck M, Neumann S, Karakesisoglou I, Noegel AA (2012) Cytoskeletal interactions at the nuclear envelope mediated by nesprins. Int $\mathrm{J}$ Cell Biol. doi:10.1155/2012/736524

67. Mislow JM, Holaska JM, Kim MS, Lee KK, Segura-Totten M, Wilson KL, McNally EM (2002) Nesprin-1 alpha self-associates and binds directly to emerin and lamin $\mathrm{A}$ in vitro. FEBS Lett 525:135-140

68. Zhou Z, Du X, Cai Z, Song X, Zhang H, Mizuno T, Suzuki E, Yee MR, Berezov A, Murali R, Wu SL, Karger BL, Greene MI, Wang Q (2012) Structure of Sad1-UNC84 homology (SUN) domain defines features of molecular bridge in nuclear envelope. J Biol Chem 287:5317-5326

69. Gomes ER, Jani S, Gundersen GG (2005) Nuclear movement regulated by $\mathrm{Cdc} 42$, MRCK, myosin, and actin flow establishes MTOC polarization in migrating cells. Cell 121:451-463 